Interfaces and Free Boundaries 14 (2012), 205-230

DOI 10.4171/IFB/280

\title{
Hele-Shaw flow in thin threads: A rigorous limit result
}

\author{
Bogdan-VASILE MATIOC \\ Institute of Applied Mathematics, Leibniz University Hannover, Hannover, Germany \\ E-mail:matioc@ifam.uni-hannover.de \\ GEORg PROKERT \\ Faculty of Mathematics and Computing Science, Technical University Eindhoven, Eindhoven, The \\ Netherlands \\ E-mail: g.prokert@tue.nl
}

[Received 5 April 2011 and in revised form 21 February 2012]

\begin{abstract}
We rigorously prove the convergence of appropriately scaled solutions of the 2D Hele-Shaw moving boundary problem with surface tension in the limit of thin threads to the solution of the formally corresponding Thin Film equation. The proof is based on scaled parabolic estimates for the nonlocal, nonlinear evolution equations that arise from these problems.
\end{abstract}

2010 Mathematics Subject Classification: Primary 35R37; Secondary 76D27, 76D08.

Keywords: Hele-Shaw flow, surface tension, Thin Film equation, degenerate parabolic equation

\section{Introduction and main result}

In theoretical fluid mechanics, the investigation of limit cases in which the thickness of the flow domain is small compared to its other lengthscale(s) is a classical subject. In most situations, the simplified equations describing these limit cases are derived formally from the original problem by expansion with respect to the small parameter describing the ratio of the lengthscales. Although lots of work have been devoted to the investigation of the limit equations (lubrication equations or various so-called Thin Film equations), the question of justifying the approximation by comparing the solutions of the original problem to those of the corresponding limit problem is less studied. This is true in particular when a moving boundary is an essential part of the original problem.

In the case of 2D Hele-Shaw flow in a thin layer, the only rigorous limit result known to us has been proved by Giacomelli and Otto [9]. Their approach is based on variational methods and can even handle degenerate cases and complicated geometries. However, the existence of global smooth solutions of the Hele-Shaw problem under consideration has to be presupposed, and the obtained result on the closeness to some solution of the Thin Film equation is in a relatively weak sense and technically rather complicated.

It is the aim of the present paper to provide a justification of the same limit equation using quite different, more standard methods. Starting from a strictly positive solution of the Thin Film equation, we prove the solvability of the corresponding moving boundary problems for large times. If the initial shape is smooth, approximations to arbitrary order and in arbitrarily strong norms are obtained. Moreover, our approach is straightforwardly generalizable to a multidimensional setting. However, it is restricted to the nondegenerate case of strictly positive film thickness and to a simple layer geometry. 


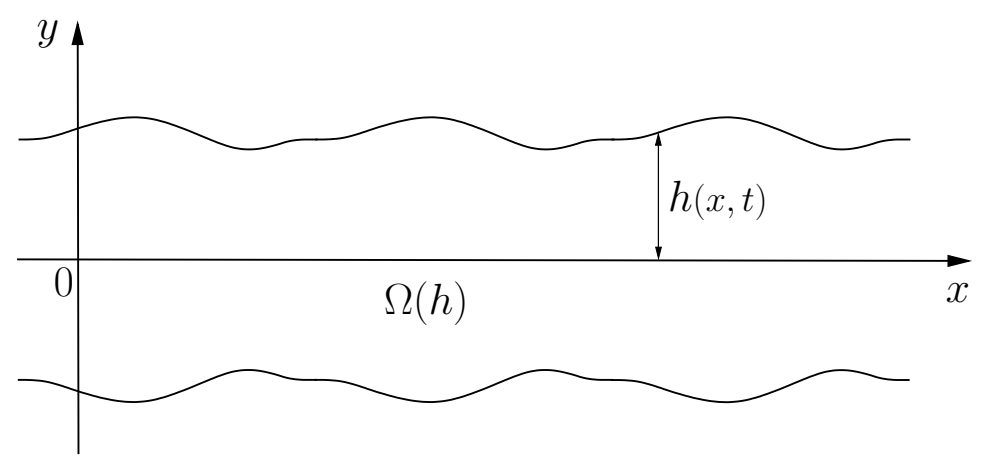

FIG. 1. The considered geometry and basic notation

More precisely, we consider 2D Hele-Shaw flow in a periodic, thin liquid domain (i.e., a thread), symmetric about the $x$-axis, with surface tension as sole driving mechanism for the flow (see Fig. 1).

This problem (in the unscaled version, with surface tension coefficient normalized to 1) consists in finding a positive function $h \in C^{1}\left([0, T], C^{2}(\mathbb{S})\right), \mathbb{S}=\mathbb{R} /[0,2 \pi)$, and, for each $t \in[0, T]$, a function $u$ defined on

$$
\Omega(h):=\{(x, y) \in \mathbb{S} \times \mathbb{R}|| y \mid<h(x)\}
$$

such that

$$
\left\{\begin{aligned}
-\Delta u & =0 & & \text { in } \Omega(h), \\
u & =-\kappa(h) & & \text { on } \partial \Omega(h), \\
\partial_{t} h+\nabla u \cdot\left(-h^{\prime}, 1\right)^{\top} & =0 & & \text { on } \Gamma_{+}(h),
\end{aligned}\right.
$$

where $\Gamma_{+}(h):=\{(x, h(x)) \mid x \in \mathbb{S}\}$, and

$$
\kappa(h):=\frac{h^{\prime \prime}}{\left(1+\left(h^{\prime}\right)^{2}\right)^{3 / 2}}
$$

is the curvature of $\Gamma_{+}(h)$. (Here and in the sequel, for the sake of brevity we write $h$ instead of $h(t)$ or $h(\cdot, t)$ when no confusion seems likely. Moreover, we identify functions on $\mathbb{S}$ with functions on $\partial \Omega$ using the pull-back along $x \mapsto(x, \pm h(x))$.) Note that $u$ represents the normalized pressure in the Hele-Shaw cell, and, in view of Darcy's law, the first and third equation are the incompressibility condition and the usual kinematic free boundary condition. Moreover, a uniqueness argument shows that $u$ is symmetric with respect to $y$, i.e., $u(x, y)=u(x,-y)$ for all $(x, y) \in \Omega(h)$, meaning that $u_{y}=0$ on $\mathbb{S} \times\{0\}$. Thus, this setting corresponds to the case when the bottom of the Hele-Shaw cell, which we take as being $\mathbb{S} \times\{0\}$, is impermeable. We refrain from discussing further modelling aspects and refer instead to the extensive literature on the subject, see, e.g., [4, Ch. 1]. Wellposedness results for (1.1) (in slightly different geometric settings and various classes of functions) have been proved in, e.g., $[1,8,13]$ and in $[5,6]$ for non-Newtonian fluids.

To consider thin threads we introduce a scaling parameter $\varepsilon, 0<\varepsilon \ll 1$, and rescale by

$$
x=\tilde{x}, \quad y=\varepsilon \tilde{y}, \quad h=\varepsilon \tilde{h}, \quad \tilde{u}(\tilde{x}, \tilde{y})=u(\tilde{x}, \varepsilon \tilde{y}) .
$$


Then $\tilde{u}$ is defined on $\Omega(\tilde{h})$, and $(\tilde{h}, \tilde{u})$ satisfies the $\varepsilon$-dependent problem

$$
\left\{\begin{aligned}
-\varepsilon^{2} \tilde{u}_{\tilde{x} \tilde{x}}-\tilde{u}_{\tilde{y} \tilde{y}} & =0 & & \text { in } \Omega(\tilde{h}), \\
\tilde{u} & =-\varepsilon \kappa(\varepsilon, \tilde{h}) & & \text { on } \partial \Omega(\tilde{h}), \\
\partial_{t} \tilde{h}+\nabla_{(\tilde{x}, \tilde{y})} \tilde{u} \cdot\left(-\tilde{h}^{\prime}, \varepsilon^{-2}\right)^{\top} & =0 & & \text { on } \Gamma_{+}(\tilde{h}),
\end{aligned}\right.
$$

where

$$
\kappa(\varepsilon, \tilde{h}):=\frac{\tilde{h}^{\prime \prime}}{\left(1+\varepsilon^{2}\left(\tilde{h}^{\prime}\right)^{2}\right)^{3 / 2}} .
$$

Expanding $\tilde{u}$ and $\kappa_{\varepsilon}$ formally in power series in $\varepsilon$ we obtain

$$
\tilde{u}=\varepsilon u_{1}+\varepsilon^{3} u_{3}+O\left(\varepsilon^{4}\right),
$$

where in particular

$$
\left(u_{1}\right)_{\tilde{x}}=-\tilde{h}^{\prime \prime \prime}, \quad\left(u_{3}\right)_{\tilde{y}}=\tilde{h} \tilde{h}^{\prime \prime \prime \prime} \quad \text { on } \Gamma_{+}(\tilde{h})
$$

So

$$
\partial_{t} \tilde{h}+\varepsilon\left(\tilde{h} \tilde{h}^{\prime \prime \prime}\right)^{\prime}=O\left(\varepsilon^{2}\right),
$$

and after rescaling $t=\varepsilon^{-1} \tilde{t}$, suppressing tildes and neglecting higher order terms we obtain the well-known Thin Film equation

$$
\partial_{t} h_{0}+\left(h_{0} h_{0}^{\prime \prime \prime}\right)^{\prime}=0 \quad \text { on } \mathbb{S} .
$$

Observe that (1.3) is a fourth order parabolic equation for positive $h_{0}$ which degenerates as $h_{0}$ approaches 0 . For a review of the extensive literature on this and related equations we refer to [11]. In the modelling context discussed here, $(1.3)$ is used in $[2,3]$ to study the breakup behavior of Hele-Shaw threads. Note that (1.3) and its multidimensional analogue

$$
\partial_{t} h+\operatorname{div}(h \nabla \Delta h)=0,
$$

also appear in models of ground water flow [11].

In view of the time rescaling, $h_{0}$ should be an approximation to

$$
h_{\varepsilon}:=\left[t \mapsto \tilde{h}\left(\varepsilon^{-1} t\right)\right],
$$

where $\tilde{h}$ solves (1.2), i.e., $h_{\varepsilon}$ solves (with an appropriate $v$ and omitting tildes)

$$
\left\{\begin{aligned}
-\varepsilon^{2} v_{x x}-v_{y y} & =0 & & \text { in } \Omega\left(h_{\varepsilon}\right), \\
v & =-\kappa\left(\varepsilon, h_{\varepsilon}\right) & & \text { on } \partial \Omega\left(h_{\varepsilon}\right), \\
\partial_{t} h_{\varepsilon}+\nabla v \cdot\left(-h_{\varepsilon}^{\prime}, \varepsilon^{-2}\right)^{\top} & =0 & & \text { on } \Gamma_{+}\left(h_{\varepsilon}\right) .
\end{aligned}\right.
$$

(Of course, (1.5) can be obtained immediately from (1.1) by choosing the "correct" scaling $u=\varepsilon \tilde{u}$, $t=\varepsilon^{-1} \tilde{t}$ at once. That scaling, however, is in itself rather a result of the above calculations.)

Our rigorous justification of the Thin Film approximation for (1.1) will therefore consist in showing that for any positive initial datum $h^{*}$ (from a suitable class of functions), the common initial condition

$$
h_{0}(0)=h_{\varepsilon}(0)=h^{*}
$$


implies existence and uniqueness of solutions to (1.3) and (1.5) (for all sufficiently small $\varepsilon$ ) on the same time interval, and

$$
h_{\varepsilon} \rightarrow h_{0} \quad \text { as } \varepsilon \downarrow 0
$$

(in a suitable sense). This will be made precise in our main result Theorem 1.2 below. Observe that this, in particular, implies that the existence time in the original timescale, i.e., for solutions of (1.2), goes to infinity as $\varepsilon$ becomes small.

We will make use of the following preliminary, nonuniform well-posedness result for solutions to (1.5). It is not optimal with respect to the demanded regularity but this is not our concern here.

THEOREM 1.1 Let $s \geqslant 7$ be an integer and $h^{*} \in H^{s+1}(\mathbb{S})$ a positive function. Then we have:

(i) Existence and uniqueness: Problem (1.5) with initial condition $h_{\varepsilon}(0)=h^{*}$ has a unique maximal solution

$$
h_{\varepsilon} \in C\left(\left[0, T_{\varepsilon}\right), H^{s}(\mathbb{S})\right) \cap C^{1}\left(\left[0, T_{\varepsilon}\right), H^{s-3}(\mathbb{S})\right)
$$

for some $T_{\varepsilon}=T_{\varepsilon}\left(h^{*}\right) \in(0, \infty]$.

(ii) Analyticity: We have

$$
\left.\left[(x, t) \mapsto h_{\varepsilon}(x, t)\right]\right|_{\mathbb{S} \times\left(0, T_{\varepsilon}\right)} \in C^{\omega}\left(\mathbb{S} \times\left(0, T_{\varepsilon}\right)\right) .
$$

(iii) Blowup: If $T_{\varepsilon}<\infty$ then

$$
\liminf _{t \uparrow T_{\varepsilon}}\left(\min _{x \in \mathbb{S}} h_{\varepsilon}(x, t)\right)=0 \quad \text { or } \quad \limsup _{t \uparrow T_{\varepsilon}}\left\|h_{\varepsilon}(\cdot, t)\right\|_{H^{s}(S)}=\infty .
$$

Existence and uniqueness of the solution on some time interval $\left[0, \Theta_{\varepsilon}\left(h^{*}\right)\right]$ can be proved by parabolic energy estimates and Galerkin approximations as in [13] (for a different geometry). The arguments given there can also be used to prove (iii) by a standard continuation argument. More precisely, for initial values $h^{*}$ that satisfy $h^{*}>\alpha>0$ and $\left\|h^{*}\right\|_{s} \leqslant M$ with any positive constants $\alpha$ and $M$, the existence time $\Theta_{\varepsilon}\left(h^{*}\right)$ has a positive lower bound depending only on these constants. This implies the blowup result (iii). For a proof of (ii) we refer to the framework given in [7] which is applicable here as well. Essentially, analyticity follows from the analytic character of all occurring nonlinearities together with the translational invariance of the problem.

We are going to state the main result now. It sharpens (1.6) as it also gives the asymptotics of $h_{\varepsilon}$ to arbitrary order $n \in \mathbb{N}$. This is achieved by imposing strong smoothness demands on the initial value (or correspondingly, on $h_{0}$ ). To avoid additional technicalities, we have not strived for optimal regularity results.

THEOREM 1.2 Let $s, n \in \mathbb{N}, s \geqslant 10$ be given. There is an integer $\beta=\beta(s, n) \in \mathbb{N}$ such that for any positive solution

$$
h_{0} \in C\left([0, T], H^{\beta}(\mathbb{S})\right) \cap C^{1}\left([0, T], H^{\beta-4}(\mathbb{S})\right)
$$

of (1.3) there are $\varepsilon_{0}=\varepsilon_{0}\left(s, n, h_{0}\right)>0, C=C\left(s, n, h_{0}\right)$ and functions

$$
h_{1}, \ldots h_{n-1} \in C\left([0, T], H^{s}(\mathbb{S})\right)
$$

depending on $h_{0}$ only such that for all $\varepsilon \in\left(0, \varepsilon_{0}\right)$

(i) Problem (1.5) with initial condition $h_{\varepsilon}(0)=h_{0}(0)$ has precisely one solution

$$
h_{\varepsilon} \in C\left([0, T], H^{\beta-1}(\mathbb{S})\right) \cap C^{1}\left([0, T], H^{\beta-4}(\mathbb{S})\right),
$$


(ii)

$$
\left\|h_{\varepsilon}-\left(h_{0}+\varepsilon h_{1}+\ldots+\varepsilon^{n-1} h_{n-1}\right)\right\|_{C\left([0, T], H^{s}(\mathbb{S})\right)} \leqslant C \varepsilon^{n} .
$$

As expected, the functions $h_{1}, h_{2}, \ldots$ satisfy the (linear parabolic) equations arising from formal expansion with respect to $\varepsilon$. For details, see Lemma 4.2 below.

Both this theorem and its proof are in strong analogy to [10], where the parallel problem for Stokes flow in a thin layer has been discussed. In this case, the limit equation, also called a Thin Film equation, is

$$
\partial_{t} h+\frac{1}{3} \operatorname{div}\left(h^{3} \nabla \Delta h\right)=0 .
$$

The remainder of this paper is devoted to the proof of Theorem 1.2. For this purpose, we first transform (1.5) to a fixed domain and rewrite the problem as a nonlinear, nonlocal operator equation for $h_{\varepsilon}$. Some scaled estimates are gathered in Section 2. In Section 3 we discuss estimates for our nonlinear operators, while Section 4 provides the necessary details on the series expansions that are used. Finally, the proof of Theorem 1.2 is completed in Section 5.

Technically, the main difficulty in comparison with the unscaled problem is the fact that the elliptic estimates for the (scaled) Laplacian and related operators degenerate as $\varepsilon$ becomes small. To handle this, weighted norms are introduced, and estimates in such norms have to be proved. In particular, the coercivity estimate for the transformed and scaled Dirichlet-Neumann operator given in Lemma 3.3 will be pivotal. On the other hand, the loss of regularity can be compensated by higher order expansions and interpolation. Moreover, as in [10], the ellipticity of the curvature operator is crucial. Therefore, the corresponding problems with gravity instead of surface tension appear intractable by the approach used here.

\section{Scaled trace inequalities and an extension operator}

In the remainder of this paper we let $\Gamma_{ \pm}:=\mathbb{S} \times\{ \pm 1\}$ denote the boundary components of our fixed reference domain $\Omega:=\mathbb{S} \times(-1,1)$. We write $H^{s}(\Omega), H^{s}\left(\Gamma_{ \pm}\right)$for the usual $L^{2}$-based Sobolev spaces of order $s \in \mathbb{R}$, while by definition $H^{s}(\partial \Omega):=H^{s}\left(\Gamma_{+}\right) \times H^{s}\left(\Gamma_{-}\right)$. Functions $f \in H^{s}\left(\Gamma_{ \pm}\right)$ may be represented by their Fourier series expansions

$$
f(x, \pm 1)=\sum_{p \in \mathbb{Z}} \widehat{f}(p) e^{i p x}, \quad x \in \mathbb{S},
$$

with $\widehat{f}(p)$ the $p$-th Fourier coefficient of $f$. Consequently, the norm of $f$ may be defined by the relation

$$
\|f\|_{s}^{\Gamma_{ \pm}}:=\left(\sum_{p \in \mathbb{Z}}\left(1+|p|^{2}\right)^{s}|\widehat{f}(p)|^{2}\right)^{1 / 2} .
$$

Similarly, functions $w \in H^{s}(\Omega)$ may be written in terms of their Fourier series

$$
w(x, y)=\sum_{p \in \mathbb{Z}} w_{p}(y) e^{i p x}, \quad(x, y) \in \Omega,
$$

and the norm of $w$ is given, for $s \geqslant 0$, by the following expression:

$$
\|w\|_{s}^{\Omega}:=\left(\sum_{p \in \mathbb{Z}}\left(\left\|w_{p}\right\|_{s}^{I}\right)^{2}+|p|^{2 s}\left(\left\|w_{p}\right\|_{0}^{I}\right)^{2}\right)^{1 / 2},
$$


where $I:=(-1,1)$. Given $\varepsilon \in(0,1)$ and $s \geqslant 1$, we introduce the following scaled norms on $H^{s}(\Omega)$

$$
\|w\|_{s, \varepsilon}^{\Omega}:=\|w\|_{s-1}^{\Omega}+\left\|\partial_{2} w\right\|_{s-1}^{\Omega}+\varepsilon\|w\|_{s}^{\Omega}, \quad w \in H^{s}(\Omega),
$$

which are equivalent to the standard Sobolev norm $\|\cdot\|_{s}^{\Omega}$ but, for $\varepsilon \rightarrow 0$, degenerate to a weaker norm. The scaling is indicated by the first equation in (1.5), and the scaled norms will enable us to take into account the different behaviour of the partial derivatives of the function $v$ in system (1.5) with respect to $\varepsilon$ when $\varepsilon \rightarrow 0$. To do this we first introduce an appropriate extension operator for functions $f \in H^{s}(\partial \Omega)$ and reconsider some classical estimates in the weighted norms.

Given $\varepsilon \in(0,1)$, we set for simplicity

$$
\nabla_{\varepsilon}:=\left(\partial_{1, \varepsilon}, \partial_{2, \varepsilon}\right):=\left(\varepsilon \partial_{1}, \partial_{2}\right)
$$

Lemma 2.1 (a) There exists a positive constant $C$ such that for all $\varepsilon \in(0,1)$ and $w \in H^{1}(\Omega)$ the following Poincaré's inequalities are satisfied:

$$
\begin{gathered}
\|w\|_{0}^{\Omega} \leqslant C\left\|\nabla_{\varepsilon} w\right\|_{0}^{\Omega} \quad \text { if }\left.\quad w\right|_{\partial \Omega}=0, \\
\|w\|_{0}^{\Omega} \leqslant C \varepsilon^{-1}\left\|\nabla_{\varepsilon} w\right\|_{0}^{\Omega} \quad \text { if } \quad \int_{\Gamma_{+}} w d \sigma=0 .
\end{gathered}
$$

(b) There exists a positive constant $C$ such that the trace inequality

$$
\|w\|_{t}^{\partial \Omega}+\sqrt{\varepsilon}\|w\|_{t+1 / 2}^{\partial \Omega} \leqslant C\|w\|_{t+1, \varepsilon}^{\Omega}
$$

is satisfied by all $\varepsilon \in(0,1), t \in \mathbb{N}$, and $w \in H^{t+1}(\Omega)$.

(c) Given $\varepsilon \in(0,1)$, there exists linear extension operators $E_{ \pm}: H^{t+1 / 2}\left(\Gamma_{ \pm}\right) \longrightarrow H^{t+1}(\Omega)$ such that $\left.\left(E_{ \pm} f\right)\right|_{\Gamma_{ \pm}}=f, E_{ \pm} f$ are even with respect to $y$ and $E_{ \pm}$satisfy the estimates

$$
\begin{aligned}
\left\|E_{ \pm} f\right\|_{t+1, \varepsilon}^{\Omega} & \leqslant C_{t}\left(\|f\|_{t}^{\Gamma_{ \pm}}+\varepsilon^{1 / 2}\|f\|_{t+1 / 2}^{\Gamma_{ \pm}}\right), \\
\left\|\partial_{2} E_{ \pm} f\right\|_{-1 / 2} & \leqslant C\left(\|f\|_{-1 / 2}^{\Gamma_{ \pm}}+\varepsilon^{1 / 2}\|f\|_{1 / 2}^{\Gamma_{ \pm}}\right),
\end{aligned}
$$

$t \in \mathbb{N}, f \in H^{t+1 / 2}\left(\Gamma_{ \pm}\right)$, with constants independent of $\varepsilon$.

Proof. The proof of (a) is standard while that of (b) is similar to that of [10, Lemma 3.1]. To show (c), set for $f(x, 1)=\sum_{p} \widehat{f}(p) e^{i p x}$

$$
E_{+} f(x, y):=\sum_{p} w_{p}(y) e^{i p x}, \quad w_{p}(y):=y^{2} e^{i|p|\left(y^{2}-1\right)} \widehat{f}(p) .
$$

Then $\left\|w_{p}\right\|_{0}^{I} \leqslant|\widehat{f}(p)|$ and for $p \neq 0$

$$
\left\|w_{p}\right\|_{0}^{I^{2}}=|\widehat{f}(p)|^{2} \int_{-1}^{1}\left|y^{4}\right| e^{2 \varepsilon|p|\left(y^{2}-1\right)} d y \leqslant 2|\widehat{f}(p)|^{2} \int_{0}^{1} y e^{2 \varepsilon|p|\left(y^{2}-1\right)} d y \leqslant \frac{|\widehat{f}(p)|^{2}}{2 \varepsilon|p|} .
$$

Similarly, for $k \in \mathbb{N}$,

$$
\left\|w_{p}^{(k)}\right\|_{0}^{I^{2}} \leqslant C_{k}\left(1+(\varepsilon|p|)^{2 k-1}\right)|\widehat{f}(p)|^{2} \leqslant C_{k}\left(1+|p|^{2 k-1}\right)|\widehat{f}(p)|^{2} .
$$


Consequently,

$$
\begin{aligned}
& \left\|E_{+} f\right\|_{t}^{\Omega^{2}} \leqslant \sum_{p}\left[\left\|w_{p}\right\|_{t}^{I^{2}}+|p|^{2 t}\left\|w_{p}\right\|_{0}^{I^{2}}\right] \leqslant C\|f\|_{t}^{\Gamma_{+}{ }^{2}}, \\
& \left\|\partial_{2} E_{+} f\right\|_{t+1}^{\Omega}{ }^{2} \leqslant \sum_{p}\left[\left\|w_{p}^{\prime}\right\|_{t}^{I^{2}}+|p|^{2 t}\left\|w_{p}^{\prime}\right\|_{0}^{I^{2}}\right] \leqslant C\left(\|f\|_{t}^{\Gamma_{+}{ }^{2}}+\varepsilon\|f\|_{t+1 / 2}^{\Gamma_{+}}{ }^{2}\right), \\
& \varepsilon^{2}\left\|E_{+} f\right\|_{t+1}^{\Omega}{ }^{2} \leqslant \varepsilon^{2} \sum_{p}\left[\left\|w_{p}\right\|_{t+1}^{I}{ }^{2}+|p|^{2 t+2}\left\|w_{p}\right\|_{0}^{I^{2}}\right] \leqslant C \varepsilon\|f\|_{t+1 / 2}^{\Gamma_{+}}{ }^{2} .
\end{aligned}
$$

This proves (2.4). To verify (2.5), note that

$$
\left\|\partial_{2} E_{+} f\right\|_{-1 / 2}^{\Gamma_{+}}{ }^{2}=\sum_{p}\left(1+|p|^{2}\right)^{-1 / 2}\left|w_{p}^{\prime}(1)\right|^{2}
$$

and

$$
\left|w_{p}^{\prime}(1)\right|^{2} \leqslant C\left(1+\varepsilon^{2}|p|^{2}\right)|\widehat{f}(p)|^{2}
$$

This implies the result for $E_{+}$, the construction for $E_{-}$is analogous.

Choosing an appropriate smooth cutoff function, one can construct a linear extension operator $E: H^{t+1 / 2}(\partial \Omega) \longrightarrow H^{t+1}(\Omega)$ such that $\left.(E f)\right|_{\partial \Omega}=f, E f$ is even with respect to the variable $y$ if $f(\cdot,-1)=f(\cdot, 1)$, and $E$ satisfies the estimates

$$
\begin{aligned}
\|E f\|_{t+1, \varepsilon}^{\Omega} & \leqslant C_{t}\left(\|f\|_{t}^{\partial \Omega}+\varepsilon^{1 / 2}\|f\|_{t+1 / 2}^{\partial \Omega}\right), \\
\left\|\partial_{2} E f\right\|_{-1 / 2}^{\partial \Omega} & \leqslant C\left(\|f\|_{-1 / 2}^{\partial \Omega}+\varepsilon^{1 / 2}\|f\|_{1 / 2}^{\partial \Omega}\right),
\end{aligned}
$$

$t \in \mathbb{N}, f \in H^{t+1 / 2}(\partial \Omega)$, with constants independent of $\varepsilon$.

\section{Uniform estimates for the scaled and transformed Dirichlet problem}

In this section we prove uniform estimates for the solution of the Dirichlet problem consisting of the first two equations of (1.5), by using the scaled norms defined above. To this end we first transform the problem (1.5) to the strip $\Omega$ by using a diffeomorphism depending on the moving boundary $h_{\varepsilon}$.

Let $m$ be $\Omega$ or $\Gamma_{ \pm}, \sigma>\operatorname{dim} m / 2, t \leqslant \sigma$. We will repeatedly and without explicit mentioning use the product estimate

$$
\left\|z_{1} z_{2}\right\|_{t}^{m} \leqslant C\left\|z_{1}\right\|_{t}^{m}\left\|z_{2}\right\|_{\sigma}^{m}, \quad z_{1} \in H^{t}(m), z_{2} \in H^{\sigma}(m) .
$$

For the remainder of the paper, let $s$ and $s_{0}$ be such that $s, s_{0}+1 / 2 \in \mathbb{N}, s_{0} \geqslant 7 / 2, s \geqslant 2 s_{0}+3$. (For example, $s_{0}=7 / 2$ and any $s \geqslant 10$ is possible, cf. Theorem 1.2.) For given $\alpha, M>0$, define the open subset $U_{s}:=U(s, M, \alpha)$ of $H^{s}(\mathbb{S})$ by

$$
U_{s}:=\left\{h \in H^{s}(\mathbb{S}):\|h\|_{s}<M \text { and } \min _{\mathbb{S}} h>\alpha\right\} .
$$

Moreover, define the (trivial) maps $\phi_{ \pm}: \Gamma_{ \pm} \longrightarrow \mathbb{S}$ by $\phi_{ \pm}(x, \pm 1)=x$.

To avoid losing regularity when transforming the problem onto the fixed reference manifold $\Omega$, we modify [10, Lemma 4.1] to obtain the following result: 
LEMMA 3.1 (Extension of $h$ ) There exists a map

$$
[h \mapsto \widetilde{h}] \in \mathcal{L}\left(H^{\sigma}(\mathbb{S}), H^{\sigma+1 / 2}(\Omega)\right), \quad \sigma>3 / 2,
$$

with the following properties:

(i) $\widetilde{h}$ is even, $\left.\widetilde{h}\right|_{\Gamma_{+}}=h \circ \phi_{+}$, and $\left.\partial_{2} \widetilde{h}\right|_{\Gamma_{+}}=0$;

(ii) If $h \in U_{s}$ and $\beta \in(0,1)$, then $\Phi_{h}:=[(x, y) \longmapsto(x, y \widetilde{h}(x, y))] \in \operatorname{Diff}^{2}(\Omega, \Omega(h))$.

In a first step we use the diffeomorphism $\Phi_{h}$ to transform the scaled problem (1.5) into a nonlinear and nonlocal evolution equation on $\mathbb{S}$, cf. (3.3). Therefore, we note that if $h_{\varepsilon}:\left[0, T_{\varepsilon}\right) \rightarrow U_{s}$ is a solution of the scaled problem (1.5), then setting $w:=-v \circ \Phi_{h_{\varepsilon}}$, we find that the pair $\left(h_{\varepsilon}, w\right)$ solves the problem

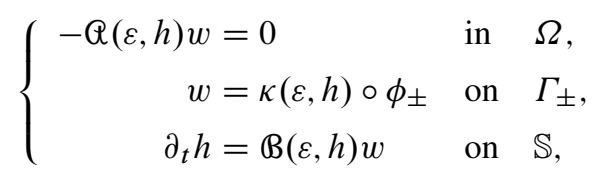

where $Q:(0,1) \times U_{s} \rightarrow \mathcal{L}\left(H^{s-3 / 2}(\Omega), H^{s-7 / 2}(\Omega)\right)$ is the linear operator given by

$$
Q(\varepsilon, h) w:=D_{i} D_{i} w
$$

with

$$
D_{1}:=\varepsilon\left(\partial_{1}+a_{1} \partial_{2}\right), \quad D_{2}:=a_{2} \partial_{2},
$$

and $a_{i}, i=1,2$ given by

$$
a_{1}:=-\frac{\partial_{1}(y \widetilde{h})}{\partial_{2}(y \widetilde{h})}, \quad a_{2}:=\frac{1}{\partial_{2}(y \widetilde{h})} .
$$

Furthermore, we define the boundary operator $\mathbb{B}:(0,1) \times \mathcal{U}_{s} \rightarrow \mathcal{L}\left(H^{s-3 / 2}(\Omega), H^{s-3}(\mathbb{S})\right)$ by the relation

$$
\begin{aligned}
\mathbb{B}(\varepsilon, h) w(x): & =\left(-h^{\prime} \partial_{1}\left(w \circ \Phi_{h}^{-1}\right)+\varepsilon^{-2} \partial_{2}\left(w \circ \Phi_{h}^{-1}\right)\right)(x, h(x)) \\
& =\left(-h^{\prime}\left(\partial_{1} w+a_{1} \partial_{2} w\right)+\varepsilon^{-2} a_{2} \partial_{2} w\right)(x, 1), \quad x \in \mathbb{S} .
\end{aligned}
$$

It is not difficult to see that $\mathbb{B}(\varepsilon, h)$ may be also written as

$$
\mathbb{B}(\varepsilon, h) w=\left.\varepsilon^{-2}\left(\frac{a_{i, \varepsilon}}{a_{2}} D_{i} w\right)\right|_{\Gamma_{+}} \circ \phi_{+}^{-1} \quad \text { on } \mathbb{S},
$$

where $a_{1, \varepsilon}:=\varepsilon a_{1}$ and $a_{2, \varepsilon}:=a_{2}$.

Given $f \in H^{s-2}(\mathbb{S})$ and $(\varepsilon, h) \in(0,1) \times U_{s}$, we denote throughout this paper by $w(\varepsilon, h)\{f\}$ the solution $w$ of the Dirichlet problem

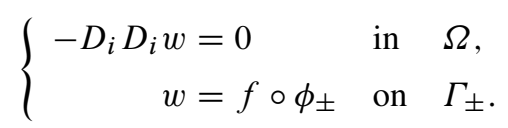

With this notation, Problem (3.1) is equivalent to the abstract evolution equation

$$
\partial_{t} h=\mathcal{F}(\varepsilon, h)
$$


where we set

$$
F(\varepsilon, h)\{f\}:=\mathbb{B}(\varepsilon, h) w(\varepsilon, h)\{f\},
$$

and the nonlinear and nonlocal operator $\mathcal{F}:(0,1) \times U_{s} \rightarrow H^{s-3}(\mathbb{S})$ is given by the relation

$$
\mathcal{F}(\varepsilon, h):=F(\varepsilon, h)\{\kappa(\varepsilon, h)\}, \quad(\varepsilon, h) \in(0,1) \times U_{s} .
$$

It will become clear from the considerations that follow that $w, F$, and $\mathcal{F}$ depend smoothly on their variables, i.e.,

$$
\begin{aligned}
& w \in C^{\infty}\left((0,1) \times u_{s}, \mathcal{L}\left(H^{s-2}(\mathbb{S}), H^{s-3 / 2}(\Omega)\right)\right), \\
& F \in C^{\infty}\left((0,1) \times u_{s}, \mathcal{L}\left(H^{s-2}(\mathbb{S}), H^{s-3}(\mathbb{S})\right)\right), \\
& \mathcal{F} \in C^{\infty}\left((0,1) \times u_{s}, H^{s-3}(\mathbb{S})\right) .
\end{aligned}
$$

We start by estimating $w$ and its derivatives, and finish the section by proving estimates for the function $F$. Some of the proofs rely on the following scaled version of the integration by parts formula

$$
\int_{\Omega} \frac{1}{a_{2}} v D_{i} w d x=-\int_{\Omega} \frac{1}{a_{2}} w D_{i} v d x+\int_{\Gamma_{+}} \frac{a_{i, \varepsilon}}{a_{2}} v w d \sigma-\int_{\Gamma_{-}} \frac{a_{i, \varepsilon}}{a_{2}} v w d \sigma,
$$

which is true for all functions $v, w \in H^{1}(\Omega)$.

In order to prove estimates for the solution operator $w$ of (3.2), we begin by analysing the solution operator corresponding to the same problem when both equations in (3.2) have a nonzero right hand side. As a first result we have:

Proposition 3.2 There exist constants $\varepsilon_{0}, C$ depending only on $u_{s}$ and $s_{0}$ such that for integer $t \in\left[1, s_{0}+1 / 2\right], f \in H^{t-1 / 2}(\mathbb{S}), f_{i} \in H^{t-1}(\Omega), i=0,1,2$, and $(\varepsilon, h) \in\left(0, \varepsilon_{0}\right) \times U_{s}$ the solution of the Dirichlet problem

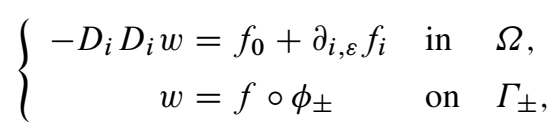

satisfies

$$
\|w\|_{t, \varepsilon}^{\Omega} \leqslant C\left(\sum_{i=0}^{2}\left\|f_{i}\right\|_{t-1}^{\Omega}+\|f\|_{t-1}+\varepsilon^{1 / 2}\|f\|_{t-1 / 2}\right) .
$$

Additionally,

$$
\left\|\partial_{2} w\right\|_{-1 / 2}^{\partial \Omega} \leqslant C\left(\sum_{i=0}^{2}\left\|f_{i}\right\|_{0}^{\Omega}+\left\|f_{2}\right\|_{-1 / 2}^{\partial \Omega}+\|f\|_{0}+\varepsilon^{1 / 2}\|f\|_{1 / 2}\right) .
$$

Proof. Step 1. We show (3.9) for $t=1$. We will consider the case $f=0$ first.

Using relation (3.7), we proceed as in [10, Lemma 3.2] and find

$$
\begin{aligned}
I & :=\int_{\Omega} \frac{1}{a_{2}} D_{i} w D_{i} w d x=-\int_{\Omega} \frac{1}{a_{2}} w D_{i} D_{i} w d x=\int_{\Omega} \frac{1}{a_{2}} w f_{0} d x+\int_{\Omega} \frac{1}{a_{2}} w \partial_{i, \varepsilon} f_{i} d x \\
& =\int_{\Omega} \frac{1}{a_{2}} w f_{0} d x-\int_{\Omega} \frac{1}{a_{2}} \partial_{i, \varepsilon} w f_{i} d x-\int_{\Omega} \partial_{i, \varepsilon}\left(\frac{1}{a_{2}}\right) w f_{i} d x,
\end{aligned}
$$


where we used integration by parts to obtain the last equality. So

$$
I \leqslant C \sum_{i=0}^{2}\left\|f_{i}\right\|_{0}^{\Omega}\|w\|_{1, \varepsilon}^{\Omega} .
$$

On the other hand,

$$
I \geqslant c \int_{\Omega}\left|\nabla_{\varepsilon} w\right|^{2}=c\left(\left\|\nabla_{\varepsilon} w\right\|_{0}^{\Omega}\right)^{2},
$$

provided $\varepsilon \in\left(0, \varepsilon_{0}\right)$ and $\varepsilon_{0}$ is sufficiently small (with a constant $c$ independent of $\varepsilon$ ). Using Poincaré's inequality (2.1), the estimate follows.

If $f \neq 0$, we let $z:=w-\widetilde{f} \in H_{-}^{t}(\Omega)$, where $\widetilde{f}:=E f$. Then $z=0$ on $\partial \Omega$ and $z$ solves in $\Omega$ the equation $-D_{i} D_{i} z=\bar{f}_{0}+\partial_{i, \varepsilon} \bar{f}_{i}$, where

$$
\bar{f}_{0}:=f_{0}-\varepsilon \partial_{2} a_{1} D_{1} \widetilde{f}-\partial_{2} a_{2} D_{2} \widetilde{f}, \quad \bar{f}_{1}=f_{1}+D_{1} \widetilde{f}, \quad \bar{f}_{2}=f_{2}+\varepsilon a_{1} D_{1} \widetilde{f}+a_{2} D_{2} \widetilde{f} .
$$

Using (2.6) and the result for homogeneous boundary data, we conclude that (3.9) holds with $t=1$.

Step 2. We show (3.10). Define

$$
\widetilde{\mathbb{B}}(\varepsilon, h) w:= \pm \varepsilon^{-2} \frac{a_{i, \varepsilon}}{a_{2}} D_{i} w \quad \text { on } \Gamma_{ \pm}
$$

and observe

$$
\partial_{2} w= \pm \varepsilon^{2} h\left(1+\varepsilon^{2} h^{\prime 2}\right)^{-1}\left(\widetilde{B}(\varepsilon, h) w+h^{\prime} \partial_{1} w\right) \quad \text { on } \Gamma_{ \pm} .
$$

We start with the case $f=0$ again. Then $\partial_{1} w=0$ and thus it is sufficient to estimate $\varepsilon^{2}\|\widetilde{B}(\varepsilon, h) w\|_{-1 / 2}^{\partial \Omega}$. For this purpose, pick $\psi \in H^{3 / 2}(\partial \Omega)$ and define $u \in H^{2}(\Omega)$ to be the solution of the Dirichlet problem

$$
\left\{\begin{aligned}
-D_{i} D_{i} u=0 & \text { in } \quad \Omega, \\
u=\psi & \text { on } \quad \partial \Omega .
\end{aligned}\right.
$$

Then, by the transformed version of Green's second identity,

$$
\begin{aligned}
\varepsilon^{2} \int_{\partial \Omega} \widetilde{\mathbb{B}}(\varepsilon, h) w \psi d \sigma & =\int_{\Omega} \frac{1}{a_{2}} D_{i} D_{i} w u, d x \\
& =-\int_{\Omega} u \frac{f_{0}+\varepsilon \partial_{1} f_{1}+\partial_{2} f_{2}}{a_{2}} d x \\
& =\int_{\Omega}\left[\varepsilon \partial_{1}\left(\frac{u}{a_{2}}\right) f_{1}+\partial_{2}\left(\frac{u}{a_{2}}\right) f_{2}-\frac{u f_{0}}{a_{2}}\right] d x \\
& +\int_{\Gamma_{-}} \frac{u}{a_{2}} f_{2} d \sigma-\int_{\Gamma_{+}} \frac{u}{a_{2}} f_{2} d \sigma .
\end{aligned}
$$

Consequently, applying the result of Step 1 to $u$,

$$
\begin{aligned}
\varepsilon^{2}\left|\int_{\partial \Omega} \widetilde{\mathbb{B}}(\varepsilon, h) w \psi d \sigma\right| & \leqslant C\left(\|u\|_{1, \varepsilon}^{\Omega} \sum_{i=0}^{2}\left\|f_{i}\right\|_{0}^{\Omega}+\left\|f_{2}\right\|_{-1 / 2}^{\partial \Omega}\|\psi\|_{1 / 2}^{\partial \Omega}\right) \\
& \leqslant C\left(\left\|f_{2}\right\|_{-1 / 2}^{\partial \Omega}+\sum_{i=0}^{2}\left\|f_{i}\right\|_{0}^{\Omega}\right)\|\psi\|_{1 / 2}^{\partial \Omega} .
\end{aligned}
$$


This implies (3.10) for $f=0$. To treat the general case, define $\tilde{f}, \bar{f}_{i}$ and $z$ as in Step 1. Then, by the preliminary result,

$$
\begin{aligned}
\left\|\partial_{2} w\right\|_{-1 / 2}^{\partial \Omega} & \leqslant\left\|\partial_{2} z\right\|_{-1 / 2}^{\partial \Omega}+\left\|\partial_{2} E f\right\|_{-1 / 2}^{\partial \Omega}, \\
\left\|\partial_{2} z\right\|_{-1 / 2}^{\partial \Omega} & \leqslant \sum_{i=0}^{2}\left\|\bar{f}_{i}\right\|_{0}^{\Omega}+\left\|\bar{f}_{2}\right\|_{-1 / 2}^{\partial \Omega}, \\
\sum_{i=0}^{2}\left\|\bar{f}_{i}\right\|_{0}^{\Omega} & \leqslant \sum_{i=0}^{2}\left\|f_{i}\right\|_{0}^{\Omega}+C\|E f\|_{1, \varepsilon}^{\Omega}, \\
\left\|\bar{f}_{2}\right\|_{-1 / 2}^{\partial \Omega} & \leqslant\left\|f_{2}\right\|_{-1 / 2}^{\partial \Omega}+C\left(\varepsilon^{2}\|f\|_{1 / 2}^{\partial \Omega}+\left\|\partial_{2} E f\right\|_{-1 / 2}^{\partial \Omega}\right),
\end{aligned}
$$

and the result follows from (2.6) and (2.7).

Step 3. We prove (3.9) by induction over $t$. The case $t=1$ has been treated in Step 1. Assume now (3.9) for an integer $t \in\left[1, s_{0}-1 / 2\right]$. Differentiating both equations of (3.8) with respect to $x$ we find that $\partial_{1} w$ satisfies

$$
\left\{\begin{aligned}
-D_{i} D_{i} \partial_{1} w & =\bar{f}_{0}+\partial_{i, \varepsilon} \overline{f_{i}} & & \text { in } \Omega \\
\partial_{1} w & =f^{\prime} \circ \phi_{ \pm} & & \text {on } \Gamma_{ \pm}
\end{aligned}\right.
$$

where

$$
\begin{aligned}
& \bar{f}_{0}=\partial_{1} f_{0}-\partial_{12} a_{i, \varepsilon} D_{i} w-\partial_{1} a_{i, \varepsilon} \partial_{2} a_{i, \varepsilon} \partial_{2} w \\
& \bar{f}_{1}=\partial_{1} f_{1}+\varepsilon \partial_{1} a_{1} \partial_{2} w \\
& \bar{f}_{2}=\partial_{1} f_{2}+\partial_{1} a_{i, \varepsilon} D_{i} w+a_{i, \varepsilon} \partial_{1} a_{i, \varepsilon} \partial_{2} w .
\end{aligned}
$$

Using this and the induction assumption, we conclude that

$$
\left\|\partial_{1} w\right\|_{t, \varepsilon}^{\Omega} \leqslant C\left(\sum_{i=0}^{2}\left\|f_{i}\right\|_{t}^{\Omega}+\|f\|_{t}+\varepsilon^{1 / 2}\|f\|_{t+1 / 2}\right) .
$$

In order to estimate $\left\|\partial_{22} w\right\|_{t-1}^{\Omega}$, we use the first equation of (3.8) and the explicit representation

$$
Q(\varepsilon, h) w:=\varepsilon^{2} \partial_{11} w+2 \varepsilon^{2} a_{1} \partial_{12} w+\left(\varepsilon^{2} a_{1}^{2}+a_{2}^{2}\right) \partial_{22} w+\left(\varepsilon^{2} \partial_{1} a_{1}+\varepsilon^{2} a_{1} \partial_{2} a_{1}+a_{2} \partial_{2} a_{2}\right) \partial_{2} w
$$

to obtain

$$
\partial_{22} w=\frac{f_{0}+\partial_{i, \varepsilon} f_{i}-\varepsilon^{2} \partial_{11} w-2 \varepsilon^{2} a_{1} \partial_{12} w-\left(\varepsilon^{2} \partial_{1} a_{1}+\varepsilon^{2} a_{1} \partial_{2} a_{1}+a_{2} \partial_{2} a_{2}\right) \partial_{2} w}{\varepsilon^{2} a_{1}^{2}+a_{2}^{2}},
$$

and see that

$$
\left\|\partial_{22} w\right\|_{t-1}^{\Omega} \leqslant C\left(\sum_{i=0}^{2}\left\|f_{i}\right\|_{t}^{\Omega}+\left\|\partial_{1} w\right\|_{t, \varepsilon}^{\Omega}+\|w\|_{t, \varepsilon}^{\Omega}\right) .
$$

Combining (3.14), (3.16), the induction assumptions, and the relation

$$
\|w\|_{t+1, \varepsilon}^{\Omega} \leqslant C\left(\|w\|_{t, \varepsilon}^{\Omega}+\left\|\partial_{1} w\right\|_{t, \varepsilon}^{\Omega}+\left\|\partial_{22} w\right\|_{t-1}^{\Omega}\right)
$$

yields the desired estimate for $\|w\|_{t+1, \varepsilon}^{\Omega}$. This completes the proof. 
Using this result we can additionally show that then

$$
\left\|\partial_{2} w(\varepsilon, h)\{f\}\right\|_{s_{0}-1 / 2}^{\Omega} \leqslant C \varepsilon^{2}\|f\|_{s_{0}+3 / 2}
$$

(Note that this involves a higher norm of $f$, but the constant involved in the estimate is of order $\varepsilon^{2}$.)

To show this, let $\phi \in H^{s-2}(\Omega)$ be the extension of $f$ given by $\phi(x, y)=f(x)$ and define $z:=w(\varepsilon, h)\{f\}-\phi$. Then

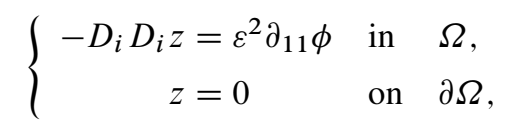

and by the unscaled trace inequality and (3.9) with $t=s_{0}+1 / 2$ we get

$$
\left\|\partial_{2} w\right\|_{s_{0}-1 / 2}^{\Omega}=\left\|\partial_{2} z\right\|_{s_{0}-1 / 2}^{\Omega} \leqslant C\|z\|_{s_{0}+1 / 2, \varepsilon}^{\Omega} \leqslant C \varepsilon^{2}\left\|\partial_{11} \phi\right\|_{s_{0}-1 / 2}^{\Omega}
$$

and therefore (3.17). In particular, this implies by the unscaled trace estimate

$$
\left\|\partial_{2} w(\varepsilon, h)\{f\}\right\|_{s_{0}-1}^{\partial \Omega} \leqslant C \varepsilon^{2}\|f\|_{s_{0}+3 / 2}
$$

Next, we prove a coercivity estimate for the scaled Dirichlet-Neumann operator $F(\varepsilon, h)$, $(\varepsilon, h) \in\left(0, \varepsilon_{0}\right) \times U_{s}$, which will be a key point in the proof of Theorem 1.2. Given $\varphi \in H^{1 / 2}(\mathbb{S})$ and $\varepsilon>0$, we set

$$
\|\varphi\|_{1 / 2, \varepsilon}:=\|\varphi\|_{0}+\varepsilon^{1 / 2}\|\varphi\|_{1 / 2} \text {. }
$$

LEMMA 3.3 There exists a positive constant $c$ such that for all $(\varepsilon, h) \in\left(0, \varepsilon_{0}\right) \times U_{s}$ and all $\varphi \in H^{3 / 2}(\mathbb{S})$ which satisfy

we have

$$
\int_{\mathbb{S}} \varphi d x=0
$$

$$
\langle F(\varepsilon, h)\{\varphi\} \mid \varphi\rangle_{L^{2}(\mathbb{S})} \geqslant c\|\varphi\|_{1 / 2, \varepsilon}^{2} .
$$

Proof. Let $w:=w(\varepsilon, h)\{\varphi\} \in H^{2}(\Omega)$, recall the definition of $\tilde{\mathbb{B}}(\varepsilon, h) w$ from (3.12) and observe that due to symmetry

$$
\tilde{\mathbb{B}}(\varepsilon, h) w(x, 1)=\tilde{\mathbb{B}}(\varepsilon, h) w(x,-1), \quad x \in \mathbb{S} .
$$

Using (3.7), we have

$$
\begin{aligned}
\langle F(\varepsilon, h)\{\varphi\} \mid \varphi\rangle_{L^{2}(\mathbb{S})} & =\int_{\Gamma_{+}} w \tilde{\mathbb{B}}(\varepsilon, h) w d \sigma \\
& =\varepsilon^{-2} \int_{\Gamma_{+}} \frac{a_{i, \varepsilon}}{a_{2}} w D_{i} w d \sigma \\
& =\frac{\varepsilon^{-2}}{2} \int_{\Gamma_{+}} \frac{a_{i, \varepsilon}}{a_{2}} w D_{i} w d \sigma-\frac{\varepsilon^{-2}}{2} \int_{\Gamma_{-}} \frac{a_{i, \varepsilon}}{a_{2}} w D_{i} w d \sigma \\
& =\frac{\varepsilon^{-2}}{2} \int_{\Omega} \frac{1}{a_{2}} D_{i} w D_{i} w d x \\
& \geqslant c \varepsilon^{-2} \int_{\Omega}\left|\nabla_{\varepsilon} w\right|^{2} d x=c \varepsilon^{-2}\left(\left\|\nabla_{\varepsilon} w\right\|_{0}^{\Omega}\right)^{2}
\end{aligned}
$$


cf. Proposition 3.2. From Poincarés inequality (2.2) together with (2.3) we obtain the desired estimate.

Now we prove estimates for the Fréchet derivatives of the solution $w=w(\varepsilon, h)\{f\}$ of (3.2) with respect to $h$. The results established in Proposition 3.2 will be used as basis for an induction argument.

Proposition 3.4 Given $k \in \mathbb{N}, h_{1}, \ldots, h_{k} \in H^{s}(\mathbb{S})$, and $f \in H^{s-2}(\mathbb{S})$, the Fréchet derivative $w^{(k)}:=w^{(k)}(\varepsilon, h)\left[h_{1}, \ldots, h_{k}\right]\{f\}$ satisfies

$$
\left\|w^{(k)}\right\|_{t, \varepsilon}^{\Omega} \leqslant C\left\|h_{1}\right\|_{s_{0}} \ldots\left\|h_{k}\right\|_{s_{0}}\|f\|_{t-1 / 2}
$$

for all integer $t \in\left[1, s_{0}-1 / 2\right]$. Additionally,

$$
\left\|\partial_{2} w^{(k)}\right\|_{-1 / 2}^{\partial \Omega} \leqslant C\left\|h_{1}\right\|_{s_{0}} \ldots\left\|h_{k}\right\|_{s_{0}}\|f\|_{1 / 2} .
$$

The constant $C$ depends only on $k, s_{0}$, and $U_{s}$.

Proof. We prove both estimates by induction over $k$. For $k=0$ they hold due to Proposition 3.2. Assume now (3.20), (3.21) for all Fréchet derivatives up to order $k$. Differentiating (3.2) $(k+1)$ times with respect to $h$, yields that $w^{(k+1)}$ is the solution of

$$
\left\{\begin{aligned}
-D_{i} D_{i} w^{(k+1)} & =\sum_{\sigma \in S_{k+1}} \sum_{l=0}^{k} C_{l} \partial_{h}^{l+1} Q(\varepsilon, h)\left[h_{\sigma(1)}, \ldots h_{\sigma(l+1)}\right] w^{(k-l)} & & \text { in } \Omega, \\
w^{(k+1)} & =0 & & \text { on } \partial \Omega,
\end{aligned}\right.
$$

where $w^{(k-l)}=w^{(k-l)}(\varepsilon, h)\left[h_{\sigma(l+2)}, \ldots, h_{\sigma(k+1)}\right]\{f\}$ and $S_{k+1}$ is the set of permutations of $\{1, \ldots, k+1\}$.

We are going to define functions $F_{i}$ in $\Omega$ such that the right hand side in (3.22) 1 can be written as

$$
\sum_{\sigma \in S_{k+1}} \sum_{l=0}^{k} C_{l} \partial_{h}^{l+1} Q(\varepsilon, h)\left[h_{\sigma(1)}, \ldots h_{\sigma(l+1)}\right] w^{(k-l)}=F_{0}+\partial_{i, \varepsilon} F_{i} .
$$

The functions $F_{i}$ are sums of terms to be specified below. For this purpose, we recall (3.15) and consider the Fréchet derivatives of the ocurring terms separately.

(i) When differentiating $\varepsilon^{2} \partial_{22} w$ we do not obtain any term on the right hand side of the first equation of (3.22).

(ii) The terms on the right hand side of the first equation of (3.22) which are obtained by differentiating $2 \varepsilon^{2} a_{1} \partial_{12} w$ may be written as follows:

$$
\varepsilon^{2} a_{1}^{(l+1)} \partial_{12} w^{(k-l)}=\partial_{1, \varepsilon}\left[\varepsilon a_{1}^{(l+1)} \partial_{2} w^{(k-l)}\right]-\partial_{1}\left(\varepsilon^{2} a_{1}^{(l+1)}\right) \partial_{2} w^{(k-l)},
$$

where $a_{1}^{(l+1)}:=a_{1}^{(l+1)}(h)\left[h_{\sigma(1)}, \ldots h_{\sigma(l+1)}\right]$. The last term belongs to $F_{0}$, while the one in the square brackets belongs to $F_{1}$.

(iii) When differentiating $\left(\varepsilon^{2} a_{1}^{2}+a_{2}^{2}\right) \partial_{22} w$ we obtain terms of the form

$\left(\varepsilon^{2} a_{1}^{2}+a_{2}^{2}\right)^{(l+1)} \partial_{22} w^{(k-l)}=\partial_{2, \varepsilon}\left[\left(\varepsilon^{2} a_{1}^{2}+a_{2}^{2}\right)^{(l+1)} \partial_{2} w^{(k-l)}\right]-\partial_{2}\left(\varepsilon^{2} a_{1}^{2}+a_{2}^{2}\right)^{(l+1)} \partial_{2} w^{(k-l)}$,

where $\left(\varepsilon^{2} a_{1}^{2}+a_{2}^{2}\right)^{(l+1)}:=\partial_{h}^{l+1}\left(\varepsilon^{2} a_{1}^{2}+a_{2}^{2}\right)(h)\left[h_{\sigma(1)}, \ldots h_{\sigma(l+1)}\right]$. The last term belongs to $F_{0}$ while the expression in the square brackets belongs to $F_{2}$. 
(iv) All terms corresponding to $\left(\varepsilon^{2} \partial_{1} a_{1}+\varepsilon^{2} a_{1} \partial_{2} a_{2}+a_{2} \partial_{2} a_{2}\right) \partial_{2} w$ are absorbed by $F_{0}$. Summarizing, we get

$$
\left\{\begin{aligned}
-D_{i} D_{i} w^{(k+1)} & =F_{0}+\partial_{i, \varepsilon} F_{i} & & \text { in } \Omega, \\
w^{(k+1)} & =0 & & \text { on } \partial \Omega,
\end{aligned}\right.
$$

where

$$
F_{i}=\sum_{\sigma \in S_{k+1}} \sum_{l=0}^{k} \alpha_{l i}\left[h_{\sigma(1)}, \ldots, h_{\sigma(l+1)}\right] \partial_{2} w^{(k-l)}
$$

and

$$
\alpha_{l i}\left[H_{1}, \ldots, H_{l+1}\right]=\sum_{\sigma^{\prime} \in S_{l+1}} \beta_{l i}(\varepsilon, \widetilde{h}) \partial^{\gamma_{l i, 1}} \widetilde{H}_{\sigma^{\prime}(1)} \ldots \partial^{\gamma_{l i, l+1}} \widetilde{H}_{\sigma^{\prime}(l+1)}
$$

with smooth functions $\beta_{l i}$ and $\left|\gamma_{l 0, j}\right| \in\{0,1,2\},\left|\gamma_{l 1, j}\right|,\left|\gamma_{l 2, j}\right| \in\{0,1\}$. Fixing $\sigma$ and $l$, writing $\alpha_{l i}:=\alpha_{l i}\left[h_{\sigma(1)}, \ldots, h_{\sigma(l+1)}\right]$ and using the induction assumption we estimate

$$
\begin{aligned}
\left\|\alpha_{l i} \partial_{2} w^{(k-l)}\right\|_{t-1}^{\Omega} & \leqslant C\left\|\alpha_{l i}\right\|_{s_{0}-3 / 2}^{\Omega}\left\|\partial_{2} w^{(k-l)}\right\|_{t-1}^{\Omega} \\
& \leqslant C\left\|\widetilde{h}_{\sigma(1)}\right\|_{s_{0}+1 / 2}^{\Omega} \ldots\left\|\widetilde{h}_{\sigma(l+1)}\right\|_{s_{0}+1 / 2}^{\Omega}\left\|w^{(k-l)}\right\|_{t, \varepsilon}^{\Omega} \\
& \leqslant C\left\|h_{1}\right\|_{s_{0}} \ldots\left\|h_{k+1}\right\|_{s_{0}}\|f\|_{t-1 / 2} .
\end{aligned}
$$

Thus

$$
\left\|F_{i}\right\|_{t-1}^{\Omega} \leqslant C\left\|h_{1}\right\|_{s_{0}} \ldots\left\|h_{k+1}\right\|_{s_{0}}\|f\|_{t-1 / 2}, \quad i=0,1,2,
$$

and (3.20) (with $k$ replaced by $k+1$ ) follows from (3.9).

Similarly,

$$
\begin{aligned}
\left\|\alpha_{l 2} \partial_{2} w^{(k-l)}\right\|_{-1 / 2}^{\partial \Omega} & \leqslant C\left\|\alpha_{l 2}\right\|_{s_{0}-1}^{\partial \Omega}\left\|\partial_{2} w^{(k-l)}\right\|_{-1 / 2}^{\partial \Omega} \leqslant C\left\|\alpha_{l 2}\right\|_{s_{0}-1 / 2}^{\Omega}\left\|\partial_{2} w^{(k-l)}\right\|_{-1 / 2}^{\partial \Omega} \\
& \leqslant C\left\|\widetilde{h}_{\sigma(1)}\right\|_{s_{0}+1 / 2}^{\Omega} \cdots\left\|\widetilde{h}_{\sigma(l+1)}\right\|_{s_{0}+1 / 2}^{\Omega}\left\|\partial_{2} w^{(k-l)}\right\|_{-1 / 2}^{\partial \Omega} \\
& \leqslant C\left\|h_{1}\right\|_{s_{0}} \ldots\left\|h_{k+1}\right\|_{s_{0}}\|f\|_{t-1 / 2} .
\end{aligned}
$$

Therefore

$$
\left\|F_{2}\right\|_{-1 / 2}^{\partial \Omega} \leqslant C\left\|h_{1}\right\|_{s_{0}} \ldots\left\|h_{k+1}\right\|_{s_{0}}\|f\|_{1 / 2},
$$

and (3.21) (with $k$ replaced by $k+1$ ) follows from (3.24) with $t=1$ and (3.10).

We prove now an estimate similar to (3.21) which is optimal with respect to one of the "variations" $h_{k}$ (say $h_{1}$ ). The price to pay here is a stronger norm for $f$.

PROPOSITION 3.5 Under the assumptions of Proposition 3.4 we additionally have

$$
\left\|\partial_{2} w^{(k)}\right\|_{-1 / 2} \leqslant C\left\|h_{1}\right\|_{3 / 2}\left\|h_{2}\right\|_{s_{0}} \ldots\left\|h_{k}\right\|_{s_{0}}\|f\|_{s_{0}-1} .
$$

The constant $C$ depends only on $k, s_{0}$, and $U_{s}$.

Proof. We show the more general estimate

$$
\left\|w^{(k)}\right\|_{1, \varepsilon}^{\Omega}+\left\|\partial_{2} w^{(k)}\right\|_{-1 / 2} \leqslant C\left\|h_{1}\right\|_{3 / 2}\left\|h_{2}\right\|_{s_{0}} \ldots\left\|h_{k}\right\|_{s_{0}}\|f\|_{s_{0}-1}
$$


by induction over $k$. For $k=0$ the statement is contained in Proposition 3.2. Assume now (3.25) for all derivatives up to some order $k$. We proceed as in the proof of Proposition 3.4, reconsider problem (3.23) and have to show now

$$
\left\|F_{i}\right\|_{0}^{\Omega},\left\|F_{2}\right\|_{-1 / 2}^{\partial \Omega} \leqslant C\left\|h_{1}\right\|_{3 / 2}\left\|h_{2}\right\|_{s_{0}} \ldots\left\|h_{k}\right\|_{s_{0}}\|f\|_{s_{0}-1} .
$$

For this purpose, we fix $\sigma$ and $l$ and estimate

$$
\left\|\alpha_{l i} \partial_{2} w^{(k-l)}\right\|_{0}^{\Omega},\left\|\alpha_{l 2} \partial_{2} w^{(k-l)}\right\|_{-1 / 2}^{\partial \Omega} .
$$

We have to distinguish two cases, depending on whether the argument $h_{1}$ occurs in the first or in the second factor.

Case 1: $\sigma^{-1}(1) \leqslant l+1$. Using Proposition 3.4 with $t=s_{0}-1 / 2$ we estimate

$$
\begin{aligned}
\left\|\alpha_{l i} \partial_{2} w^{(k-l)}\right\|_{0}^{\Omega} & \leqslant C\left\|\alpha_{l i}\right\|_{0}^{\Omega}\left\|\partial_{2} w^{(k-l)}\right\|_{s_{0}-3 / 2}^{\Omega} \\
& \leqslant C\left\|\widetilde{h}_{1}\right\|_{2}^{\Omega} \prod\left\|\widetilde{h}_{\sigma(j)}\right\|_{s_{0}+1 / 2}^{\Omega}\left\|w^{(k-l)}\right\|_{s_{0}-1 / 2, \varepsilon}^{\Omega} \\
& \leqslant C\left\|h_{1}\right\|_{3 / 2}\left\|h_{2}\right\|_{s_{0}} \ldots\left\|h_{k}\right\|_{s_{0}}\|f\|_{s_{0}-1} .
\end{aligned}
$$

The product is taken over $j \in\{1, \ldots, l+1\} \backslash\left\{\sigma^{-1}(1)\right\}$. Similarly,

$$
\begin{aligned}
\left\|\alpha_{l 2} \partial_{2} w^{(k-l)}\right\|_{-1 / 2}^{\partial \Omega} & \leqslant C\left\|\alpha_{l 2}\right\|_{1 / 2}^{\partial \Omega}\left\|\partial_{2} w^{(k-l)}\right\|_{s_{0}-2}^{\partial \Omega} \\
& \leqslant C\left\|\alpha_{l 2}\right\|_{1}^{\Omega}\left\|\partial_{2} w^{(k-l)}\right\|_{s_{0}-3 / 2}^{\Omega} \\
& \leqslant C\left\|\widetilde{h}_{1}\right\|_{2}^{\Omega} \prod\left\|\widetilde{h}_{\sigma(j)}\right\|_{s_{0}+1 / 2}^{\Omega}\left\|w^{(k-l)}\right\|_{s_{0}-1 / 2, \varepsilon}^{\Omega} \\
& \leqslant C\left\|h_{1}\right\|_{3 / 2}\left\|h_{2}\right\|_{s_{0}} \ldots\left\|h_{k}\right\|_{s_{0}}\|f\|_{s_{0}-1} .
\end{aligned}
$$

Case 2: $\sigma^{-1}(1) \leqslant l+1$. We apply the induction assumption and estimate

$$
\begin{aligned}
\left\|\alpha_{l i} \partial_{2} w^{(k-l)}\right\|_{0}^{\Omega} & \leqslant C\left\|\alpha_{l i}\right\|_{S_{0}-3 / 2}^{\Omega}\left\|\partial_{2} w^{(k-l)}\right\|_{0}^{\Omega} \\
& \leqslant C\left\|\widetilde{h}_{\sigma(1)}\right\|_{s_{0}+1 / 2}^{\Omega} \ldots\left\|\widetilde{h}_{\sigma(l+1)}\right\|_{s_{0}+1 / 2}^{\Omega}\left\|w^{(k-l)}\right\|_{1, \varepsilon}^{\Omega} \\
& \leqslant C\left\|h_{1}\right\|_{3 / 2}\left\|h_{2}\right\|_{s_{0}} \ldots\left\|h_{k}\right\|_{s_{0}}\|f\|_{s_{0}-1} .
\end{aligned}
$$

Similarly,

$$
\begin{aligned}
\left\|\alpha_{l 2} \partial_{2} w^{(k-l)}\right\|_{-1 / 2}^{\partial \Omega} & \leqslant C\left\|\alpha_{l 2}\right\|_{s_{0}-2}^{\partial \Omega}\left\|\partial_{2} w^{(k-l)}\right\|_{-1 / 2}^{\partial \Omega} \\
& \leqslant C\left\|\alpha_{l 2}\right\|_{s_{0}-3 / 2}^{\Omega}\left\|\partial_{2} w^{(k-l)}\right\|_{-1 / 2}^{\partial \Omega} \\
& \leqslant C\left\|\widetilde{h}_{\sigma(1)}\right\|_{s_{0}+1 / 2}^{\Omega} \ldots\left\|\widetilde{h}_{\sigma(l+1)}\right\|_{s_{0}+1 / 2}^{\Omega}\left\|w^{(k-l)}\right\|_{1, \varepsilon}^{\Omega} \\
& \leqslant C\left\|h_{1}\right\|_{3 / 2}\left\|h_{2}\right\|_{s_{0}} \ldots\left\|h_{k}\right\|_{s_{0}}\|f\|_{s_{0}-1} .
\end{aligned}
$$

The proof is completed now by carrying out the summations over $\sigma$ and $l$ and applying Proposition 3.2 to (3.23).

We recall (cf. (3.4))

$$
F(\varepsilon, h)\{f\}=\left.\frac{1}{h}\left(\varepsilon^{-2}+h^{\prime 2}\right)\left(\partial_{2} w(\varepsilon, h)\{f\}\right)\right|_{\Gamma_{+}} \circ \phi_{+}^{-1}-h^{\prime} f^{\prime} .
$$


Applying the product rule of differentiation and product estimates as above we find from this and Propositions 3.4 and 3.5

$$
\begin{aligned}
& \left\|F^{(m)}(\varepsilon, h)\left[h_{1}, \ldots h_{m}\right]\{f\}\right\|_{-1 / 2} \leqslant C \varepsilon^{-2}\left\|h_{1}\right\|_{s_{0}} \ldots\left\|h_{k}\right\|_{s_{0}}\|f\|_{1 / 2}, \\
& \left\|F^{(m)}(\varepsilon, h)\left[h_{1}, \ldots h_{m}\right]\{f\}\right\|_{-1 / 2} \leqslant C \varepsilon^{-2}\left\|h_{1}\right\|_{3 / 2} \ldots\left\|h_{k}\right\|_{s_{0}}\|f\|_{s_{0}-1}
\end{aligned}
$$

for all $m \in \mathbb{N},(\varepsilon, h) \in\left(0, \varepsilon_{0}\right) \times U_{s}, f \in H^{s-2}(\mathbb{S})$, and $h_{1}, \ldots h_{m} \in H^{s}(\mathbb{S})$. Additionally, using (3.18),

$$
\|F(\varepsilon, h)\{f\}\|_{s_{0}-1} \leqslant C\|f\|_{s_{0}+3 / 2} .
$$

In particular, we have

$$
\|\mathcal{F}(\varepsilon, h)\|_{s_{0}-1} \leqslant C\|h\|_{s_{0}+7 / 2} \leqslant C, \quad(\varepsilon, h) \in\left(0, \varepsilon_{0}\right) \times U_{s} .
$$

The constants depend only upon $u_{s}, s_{0}$, and $m$.

Moreover, we obtain:

LEMMA 3.6 Given $h_{1} \in H^{s}(\mathbb{S})$ and $f \in H^{s-2}(\mathbb{S})$, we have

$$
\left\|F^{\prime}(\varepsilon, h)\left[h_{1}\right]\{f\}\right\|_{-1 / 2} \leqslant C\left\|h_{1}\right\|_{3 / 2}\|f\|_{s_{0}+3 / 2} \text {. }
$$

Proof. For brevity we write $w^{\prime}:=w^{\prime}(\varepsilon, h)\{f\}$. Differentiating (3.2) with respect to $h$ yields

$$
\left\{\begin{aligned}
-D_{i} D_{i} w^{\prime} & =f_{0}+\partial_{i, \varepsilon} f_{i} \quad \text { in } \Omega, \\
w^{\prime} & =0 \quad \text { on } \partial \Omega
\end{aligned}\right.
$$

where

$$
\begin{aligned}
f_{0} & :=\varepsilon^{2} \partial_{h} a_{1}\left[h_{1}\right] \partial_{12} w+2^{-1} \partial_{h}\left(\varepsilon^{2} a_{1}^{2}+a_{2}^{2}\right)\left[h_{1}\right] \partial_{22} w, \\
f_{1} & :=\varepsilon \partial_{h} a_{1}\left[h_{1}\right] \partial_{2} w, \\
f_{2} & :=2^{-1} \partial_{h}\left(\varepsilon^{2} a_{1}^{2}+a_{2}^{2}\right)\left[h_{1}\right] \partial_{2} w, \\
w & :=w(\varepsilon, h)\{f\} .
\end{aligned}
$$

By (3.9) and (3.17) we have

$$
\left\|\partial_{2} w^{\prime}\right\|_{1 / 2} \leqslant\left\|w^{\prime}\right\|_{2, \varepsilon}^{\Omega} \leqslant C \sum_{i=0}^{2}\left\|f_{i}\right\|_{1}^{\Omega} \leqslant C\left\|h_{1}\right\|_{3 / 2}\left\|\partial_{2} w\right\|_{3}^{\Omega} \leqslant C \varepsilon^{2}\left\|h_{1}\right\|_{3 / 2}\|f\|_{s_{0}+3 / 2} .
$$

The result follows easily from this.

Next we give an estimate for the remainder term that occurs when curvature differences are linearized.

LEMmA 3.7 Let $\varepsilon \in(0,1)$ and $h, \bar{h} \in U_{s} \cap H^{s+3 / 2}(\mathbb{S})$. Then

$$
\left\|\partial_{x}^{s-1}(\kappa(\varepsilon, h)-\kappa(\varepsilon, \bar{h}))-\kappa^{\prime}(\varepsilon, h)\left[(h-\bar{h})^{(s-1)}\right]\right\|_{1 / 2} \leqslant C\left(1+\|\bar{h}\|_{s+3 / 2}\right)\|h-\bar{h}\|_{s+1 / 2} .
$$

The constant $C$ depends only on $U_{s}$. 
Proof. By the chain rule,

$$
\partial_{x}^{s-1} \kappa(\varepsilon, h)=\kappa^{\prime}(\varepsilon, h)\left[h^{(s-1)}\right]+\sum_{l=2}^{s-1} C_{p_{1} \ldots p_{l}} \kappa^{(l)}(\varepsilon, h)\left[h^{\left(p_{1}\right)}, \ldots, h^{\left(p_{l}\right)}\right]
$$

with $1 \leqslant p_{1} \leqslant \ldots \leqslant p_{l}$, and $p_{1}+\ldots+p_{l}=s-1$. This also holds if we replace $h$ by $\bar{h}$. We subtract these identities and obtain

$$
\begin{aligned}
& \partial_{x}^{s-1}(\kappa(\varepsilon, h)-\kappa(\varepsilon, \bar{h}))-\kappa^{\prime}(\varepsilon, h)\left[(h-\bar{h})^{(s-1)}\right] \\
& =\left(\kappa^{\prime}(\varepsilon, h)-\kappa^{\prime}(\varepsilon, \bar{h})\right)\left[\bar{h}^{(s-1)}\right] \\
& \quad+\sum_{l=2}^{s-1} C_{p_{1} \ldots p_{l}}\left(\left(\kappa^{(l)}(\varepsilon, h)-\kappa^{(l)}(\varepsilon, \bar{h})\right)\left[\bar{h}^{\left(p_{1}\right)}, \ldots, \bar{h}^{\left(p_{l}\right)}\right]\right. \\
& \quad+\sum_{j=1}^{l} \kappa^{(l)}(\varepsilon, h)\left[h^{\left(p_{1}\right)}, \ldots, h^{\left(p_{j-1}\right)},\left(h-\bar{h}^{\left(p_{j}\right)}, \bar{h}^{\left(p_{j+1}\right)}, \ldots, \bar{h}^{\left(p_{l}\right)}\right]\right)
\end{aligned}
$$

The terms on the right are estimated separately. One straightforwardly gets

$$
\left\|\kappa^{(l)}(\varepsilon, h)\left[h_{1}, \ldots, h_{l}\right]\right\|_{1 / 2} \leqslant C\left\|h_{1}\right\|_{3} \ldots\left\|h_{l-1}\right\|_{3}\left\|h_{l}\right\|_{5 / 2}
$$

for all $l \in \mathbb{N}, l \geqslant 1, h_{l} \in H^{5 / 2}(\mathbb{S})$ and $h_{1}, \ldots h_{l-1} \in H^{3}(\mathbb{S})$. Since $U_{s}$ is convex, we additionally have

$$
\begin{aligned}
\left\|\left(\kappa^{(l)}(\varepsilon, h)-\kappa^{(l)}(\varepsilon, \bar{h})\right)\left[h_{1}, \ldots, h_{l}\right]\right\|_{1 / 2} \\
\quad \leqslant \int_{0}^{1}\left\|\kappa^{(l+1)}(\varepsilon, r h+(1-r) \bar{h})\left[h-\bar{h}, h_{1}, \ldots, h_{l}\right]\right\|_{1 / 2} d r \\
\leqslant C\|h-\bar{h}\|_{3}\left\|h_{1}\right\|_{3} \ldots\left\|h_{l-1}\right\|_{3}\left\|h_{l}\right\|_{5 / 2} .
\end{aligned}
$$

Applying these estimates to all terms in (3.31) and adding them up yields the result.

Finally, we give a parallel estimate concerning the complete operator $\mathcal{F}$. Using the invariance of our problem with respect to horizontal translations we obtain, as in [10], Eq. (6.8), the "chain rule"

$$
\begin{aligned}
& \partial_{x}^{s-1} \mathcal{F}(\varepsilon, h)=F(\varepsilon, h)\left\{\partial_{x}^{s-1} \kappa(\varepsilon, h)\right\} \\
& \quad+\sum_{k \geqslant 1} C_{p_{1}, \ldots, p_{k+1}} F^{(k)}(\varepsilon, h)\left[h^{\left(p_{1}\right)}, \ldots, h^{\left(p_{k}\right)}\right]\left\{\partial_{x}^{p_{k+1}} \kappa(\varepsilon, h)\right\},
\end{aligned}
$$

$h \in U_{s}$ sufficiently smooth. The sum is taken over all $(k+1)$-tuples $\left(p_{1}, \ldots, p_{k+1}\right)$ satisfying $p_{1}+\ldots+p_{k+1}=s-1$ and $p_{1}, \ldots, p_{k} \geqslant 1$.

Lemma 3.8 Additionally to Lemma 3.7 , assume $\varepsilon \in\left(0, \varepsilon_{0}\right)$. Define

$$
P_{s}(\varepsilon, h, \bar{h}):=\partial_{x}^{s-1}(\mathcal{F}(\varepsilon, h)-\mathcal{F}(\varepsilon, \bar{h}))-F(\varepsilon, h)\left\{\kappa^{\prime}(\varepsilon, h)\left[(h-\bar{h})^{(s-1)}\right]\right\} .
$$


Then

$$
\left\|P_{S}(\varepsilon, h, \bar{h})\right\|_{-1 / 2} \leqslant C \varepsilon^{-2}\left(1+\|\bar{h}\|_{s+3 / 2}\right)\|h-\bar{h}\|_{s+1 / 2} .
$$

The constant $C$ depends only on $U_{s}$.

Proof. Observe that by density and continuity arguments, it is sufficient to show (3.34) under the additional assumption that $h$ and $\bar{h}$ are smooth. We infer from (3.33) that $P_{s}(\varepsilon, h, \bar{h})=E^{a}+E^{b}+G$, with

$$
\begin{aligned}
& E^{a}:=F(\varepsilon, h)\left\{\partial_{x}^{s-1} \kappa(\varepsilon, h)\right\}-F(\varepsilon, \bar{h})\left\{\partial_{x}^{s-1} \kappa(\varepsilon, \bar{h})\right\}-F(\varepsilon, h)\left\{\kappa^{\prime}(\varepsilon, h)\left[(h-\bar{h})^{(s-1)}\right]\right\}, \\
& E^{b}:=F^{\prime}(\varepsilon, h)\left[h^{(s-1)}\right]\{\kappa(\varepsilon, h)\}-F^{\prime}(\varepsilon, \bar{h})\left[\bar{h}^{(s-1)}\right]\{\kappa(\varepsilon, \bar{h})\},
\end{aligned}
$$

and $G:=\sum C_{p_{1}, \ldots, p_{k+1}} E_{p_{1}, \ldots, p_{k+1}}^{c}$, where the sum is taken over all tuples satisfying additionally $1 \leqslant p_{k+1} \leqslant s-2$, and $E_{p_{1}, \ldots, p_{k+1}}^{c}=: E^{c}$ is given by

$$
E^{c}:=F^{(k)}(\varepsilon, h)\left[h^{\left(p_{1}\right)}, \ldots, h^{\left(p_{k}\right)}\right]\left\{\partial_{x}^{p_{k+1}} \kappa(\varepsilon, h)\right\}-F^{(k)}(\varepsilon, \bar{h})\left[\bar{h}^{\left(p_{1}\right)}, \ldots, \bar{h}^{\left(p_{k}\right)}\right]\left\{\partial_{x}^{p_{k+1}} \kappa(\varepsilon, \bar{h})\right\} .
$$

We estimate $E^{a}$ first and write $E^{a}=E_{1}^{a}+E_{2}^{a}$, where

$$
\begin{aligned}
& E_{1}^{a}:=F(\varepsilon, h)\left\{\partial_{x}^{s-1}(\kappa(\varepsilon, h)-\kappa(\varepsilon, \bar{h}))-\kappa^{\prime}(\varepsilon, h)\left[(h-\bar{h})^{(s-1)}\right]\right\}, \\
& E_{2}^{a}:=(F(\varepsilon, h)-F(\varepsilon, \bar{h}))\left\{\partial_{x}^{s-1} \kappa(\varepsilon, \bar{h})\right\} .
\end{aligned}
$$

Invoking (3.26) (with $m=0$ ) and Lemma 3.7, we get that

$$
\begin{aligned}
\left\|E_{1}^{a}\right\|_{-1 / 2} & \leqslant C \varepsilon^{-2}\left\|\partial_{x}^{s-1}(\kappa(\varepsilon, h)-\kappa(\varepsilon, \bar{h}))-\kappa^{\prime}(\varepsilon, h)\left[(h-\bar{h})^{(s-1)}\right]\right\|_{1 / 2} \\
& \leqslant C \varepsilon^{-2}\left(1+\|\bar{h}\|_{s+3 / 2}\right)\|h-\bar{h}\|_{s+1 / 2} .
\end{aligned}
$$

In order to estimate $E_{2}^{a}$, we write

$$
E_{2}^{a}=\int_{0}^{1} F^{\prime}(\varepsilon, r h+(1-r) \bar{h})[h-\bar{h}]\left\{\partial_{x}^{s-1} \kappa(\varepsilon, \bar{h})\right\} d r,
$$

and using (3.26), with $m=1$, yields

$$
\left\|E_{2}^{a}\right\|_{-1 / 2} \leqslant \varepsilon^{-2}\|h-\bar{h}\|_{s_{0}}\|\bar{h}\|_{s+3 / 2} \leqslant C \varepsilon^{-2}\|\bar{h}\|_{s+3 / 2}\|h-\bar{h}\|_{s} .
$$

Similarly, we decompose $E^{b}=E_{1}^{b}+E_{2}^{b}+E_{3}^{b}$, where

$$
\begin{aligned}
E_{1}^{b} & :=\left(F^{\prime}(\varepsilon, h)-F^{\prime}(\varepsilon, \bar{h})\right)\left[\bar{h}^{(s-1)}\right]\{\kappa(\varepsilon, \bar{h})\} \\
& =\int_{0}^{1} F^{\prime \prime}(\varepsilon, r h+(1-r) \bar{h})\left[h-\bar{h}^{(s-1)}\right]\{\kappa(\varepsilon, \bar{h})\} d r \\
E_{2}^{b} & :=F^{\prime}(\varepsilon, h)\left[\bar{h}^{(s-1)}\right]\{\kappa(\varepsilon, h)-\kappa(\varepsilon, \bar{h})\}, \\
E_{3}^{b} & :=F^{\prime}(\varepsilon, h)\left[h^{(s-1)}-\bar{h}^{(s-1)}\right]\{\kappa(\varepsilon, h)\} .
\end{aligned}
$$


The estimate (3.27) with $m=1$ and $m=2$, respectively, yields

$$
\begin{aligned}
& \left\|E_{1}^{b}\right\|_{-1 / 2} \leqslant C \varepsilon^{-2}\|h-\bar{h}\|_{s_{0}}\left\|\bar{h}^{(s-1)}\right\|_{3 / 2}\|\kappa(\varepsilon, \bar{h})\|_{s_{0}} \leqslant C \varepsilon^{-2}\|\bar{h}\|_{s+1 / 2}\|h-\bar{h}\|_{s}, \\
& \left\|E_{2}^{b}\right\|_{-1 / 2} \leqslant C \varepsilon^{-2}\left\|\bar{h}^{(s-1)}\right\|_{3 / 2}\|\kappa(\varepsilon, h)-\kappa(\varepsilon, \bar{h})\|_{s_{0}} \leqslant C \varepsilon^{-2}\|\bar{h}\|_{s+1 / 2}\|h-\bar{h}\|_{s}, \\
& \left\|E_{3}^{b}\right\|_{-1 / 2} \leqslant C \varepsilon^{-2}\left\|h^{(s-1)}-\bar{h}^{(s-1)}\right\|_{3 / 2}\|\kappa(\varepsilon, h)\|_{s_{0}} \leqslant C \varepsilon^{-2}\|h-\bar{h}\|_{s+1 / 2} .
\end{aligned}
$$

To estimate $G$, we proceed similarly and decompose $E^{c}=E_{1}^{c}+E_{2}^{c}+E_{3}^{c}$, with

$$
\begin{aligned}
E_{1}^{c} & :=\left(F^{(k)}(\varepsilon, h)-F^{(k)}(\varepsilon, \bar{h})\right)\left[\bar{h}^{\left(p_{1}\right)}, \ldots, \bar{h}^{\left(p_{k}\right)}\right]\left\{\partial_{x}^{p_{k+1}} \kappa(\varepsilon, \bar{h})\right\} \\
& =\int_{0}^{1} F^{(k+1)}(\varepsilon, r h+(1-r) \bar{h})\left[h-\bar{h}^{\left(p_{1}\right)}, \ldots, \bar{h}^{\left(p_{k}\right)}\right]\left\{\partial_{x}^{p_{k+1}} \kappa(\varepsilon, \bar{h})\right\} d r \\
E_{2}^{c} & :=F^{(k)}(\varepsilon, h)\left[\bar{h}^{\left(p_{1}\right)}, \ldots, \bar{h}^{\left(p_{k}\right)}\right]\left\{\partial_{x}^{p_{k+1}}(\kappa(\varepsilon, h)-\kappa(\varepsilon, \bar{h}))\right\} \\
E_{3}^{c} & :=\sum_{i=1}^{k} F^{(k)}(\varepsilon, h)\left[\bar{h}^{\left(p_{1}\right)}, \ldots, \bar{h}^{\left(p_{i-1}\right)}, h^{\left(p_{i}\right)}-\bar{h}^{\left(p_{i}\right)}, h^{\left(p_{i+1}\right)}, \ldots, h^{\left(p_{k}\right)}\right]\left\{\partial_{x}^{p_{k+1}} \kappa(\varepsilon, \bar{h})\right\} .
\end{aligned}
$$

We distinguish two cases.

Case 1. Suppose first that $p_{k+1} \geqslant p_{j}$ for all $1 \leqslant j \leqslant k$. Then

$$
p_{k+1} \leqslant s-2, \quad p_{1}, \ldots, p_{k} \leqslant \frac{s-1}{2} \leqslant s-s_{0}-2,
$$

by the choice of $s$. Choosing $m=k+1$, we infer from $p_{k+1} \geqslant 1$ that $k+1 \leqslant s-1$, and together with relation (3.26) we find

$$
\left\|E_{1}^{c}\right\|_{-1 / 2} \leqslant C \varepsilon^{-2}\|h-\bar{h}\|_{s_{0}}\left\|\bar{h}^{\left(p_{1}\right)}\right\|_{s_{0}} \ldots\left\|\bar{h}^{\left(p_{k}\right)}\right\|_{s_{0}}\|\bar{h}\|_{s+1 / 2} \leqslant C \varepsilon^{-2}\|\bar{h}\|_{s+1 / 2}\|h-\bar{h}\|_{s},
$$

while, for $m=k$, the same relation implies

$$
\begin{aligned}
& \left\|E_{2}^{c}\right\|_{-1 / 2} \leqslant C \varepsilon^{-2}\|h-\bar{h}\|_{s+1 / 2}, \\
& \left\|E_{3}^{c}\right\|_{-1 / 2} \leqslant C \varepsilon^{-2}\|\bar{h}\|_{s+1 / 2}\|h-\bar{h}\|_{s} .
\end{aligned}
$$

Case 2. Due to symmetry, we only have to consider the case when $p_{1} \geqslant p_{j}$, for all $1 \leqslant j \leqslant k+1$. Then

$$
p_{1} \leqslant s-2, \quad p_{2}, \ldots, p_{k+1} \leqslant \frac{s-1}{2} \leqslant s-s_{0}-2,
$$

and (3.27) with $m=k+1$ and $m=k$, respectively, yields

$$
\left\|E_{i}^{c}\right\|_{-1 / 2} \leqslant C \varepsilon^{-2}\|h-\bar{h}\|_{s} .
$$

This completes the proof. 


\section{Approximation by power series in $\varepsilon$}

In this section we construct operators $\mathcal{F}_{k}$ and functions $t \mapsto h_{\varepsilon, k}(t)$ such that, in a sense to be made precise below,

$$
\mathcal{F}(\varepsilon, h)=\mathfrak{F}_{k}(\varepsilon, h)+O\left(\varepsilon^{k+1}\right),
$$

and $h_{\varepsilon, k}$ is an approximate solution to (3.3). Formally, the construction is by expansion with respect to $\varepsilon$ near 0 , i.e., $\mathfrak{F}_{k}(\varepsilon, h)$ and $h_{\varepsilon, k}$ are polynomials of order $k$ in $\varepsilon$. In lowest order $k=0$, we will recover the Thin Film equation (1.3). As this construction involves a loss of regularity that increases with $k$, we will have to assume higher smoothness of $h$.

Fix $k \in \mathbb{N}$ and let $s_{1} \geqslant s+k+15 / 2$, with $s$ as before. In this section, we will assume $h \in U_{s_{1}}$ and all constants in our estimates will be independent of $h$.

We start with a series expansion for $w(\varepsilon, h)\{f\}$.

LEMMA 4.1 For $p=0,1, \ldots, k+2$ there are operators

$$
w^{[p]} \in C^{\infty}\left(U_{s_{1}}, \mathcal{L}\left(H^{s_{1}-2}(\mathbb{S}), H^{s_{1}-2-p}(\Omega)\right)\right)
$$

such that

$$
\left\|w(\varepsilon, h)\{f\}-\sum_{p=0}^{k+2} \varepsilon^{p} w^{[p]}(h)\{f\}\right\|_{s+3 / 2}^{\Omega} \leqslant C \varepsilon^{k+3}\|f\|_{s_{1}-2} .
$$

In particular,

$$
w^{[0]}(h)\{f\}(x, y)=f(x), \quad w^{[2]}(h)\{f\}(x, y)=f^{\prime \prime}(x) \int_{y}^{1} \frac{\tau}{a_{2}^{2}(x, \tau)} d \tau, \quad w^{[p]}=0 \text { for } p \text { odd. }
$$

Proof. Recalling (3.15) we have $Q(\varepsilon, h)=\delta_{0}(h)+\varepsilon^{2} S_{2}(h)$ with

$$
\begin{aligned}
& \delta_{0}(h):=a_{2}^{2} \partial_{22}+a_{2} a_{2,2} \partial_{2}, \\
& \delta_{2}(h):=\partial_{11}^{2}+2 a_{1} \partial_{12}^{2}+a_{1}^{2} \partial_{22}^{2}+\left(a_{1,1}+a_{1} a_{1,2}\right) \partial_{2} .
\end{aligned}
$$

The terms $w^{[p]}(h)\{f\}$ are determined successively from inserting the ansatz

$$
w(\varepsilon, h)\{f\}=\sum_{p=0}^{k+2} \varepsilon^{p} w^{[p]}(h)\{f\}+R
$$

into

$$
\left\{\begin{aligned}
\left(S_{0}(h)+\varepsilon^{2} S_{2}(h)\right) w(\varepsilon, h)\{f\} & =0 & & \text { in } \Omega, \\
w(\varepsilon, h)\{f\} & =f \circ \phi_{ \pm} & & \text {on } \Gamma_{ \pm},
\end{aligned}\right.
$$

and equating terms with equal powers of $\varepsilon$. Thus we obtain

$$
\left\{\begin{array} { r l r } 
{ \delta _ { 0 } ( h ) w ^ { [ 0 ] } = 0 } & { \text { in } } & { \Omega , } \\
{ w ^ { [ 0 ] } = f \circ \phi _ { \pm } } & { \text { on } } & { \Gamma _ { \pm } }
\end{array} \quad \left\{\begin{array}{rlrl}
\delta_{0}(h) w^{[1]}=0 & \text { in } \quad & \Omega \\
w^{[1]}=0 & \text { on } & \partial \Omega .
\end{array}\right.\right.
$$

and further

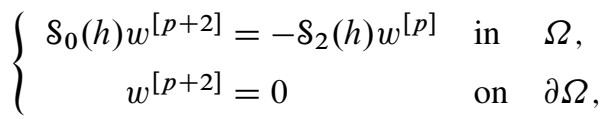


$p=0, \ldots, k$. Observe that the general problem

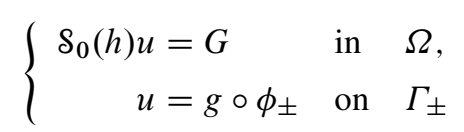

(with $g$ and $G$ even) is solved by

$$
u(x, y)=g(x)-\int_{y}^{1} \frac{1}{a_{2}(x, \tau)^{2}} \int_{0}^{\tau} G(x, s) d s d \tau,
$$

and for this solution we have

$$
\|u\|_{t}^{\Omega} \leqslant C\left(\|g\|_{t}+\|G\|_{t}^{\Omega}\right),
$$

$t \in\left[s+3 / 2, s_{1}-2\right]$. All statements concerning the mapping properties and the explicit form of the $w^{[p]}$ follow from this. To estimate the remainder, observe that

$$
\left\{\begin{aligned}
\mathbb{Q}(\varepsilon, h) R & =-\varepsilon^{k+3} S_{2}(h) w^{[k+1]}(h)\{f\}-\varepsilon^{k+4} \delta_{2}(h) w^{[k+2]}(h)\{f\} & & \text { in } \Omega, \\
R & =0 & & \text { on } \partial \Omega .
\end{aligned}\right.
$$

The estimate follows from Proposition 3.2 with $s$ replaced by $s_{1}$ and $t=s+5 / 2$.

Recall, furthermore, that

$$
\mathbb{B}(\varepsilon, h)=\varepsilon^{-2} \mathbb{B}^{[0]}(h)+\mathbb{B}^{[2]}(h)
$$

where

$$
\mathbb{B}^{[0]}, \mathbb{B}^{[2]} \in C^{\infty}\left(u_{s_{1}}, \mathcal{L}\left(H^{s+3 / 2}(\Omega), H^{s}(\mathbb{S})\right)\right)
$$

are given by

$\mathbb{B}^{[0]}(h) w=\left.h^{-1}\left(\partial_{2} w\right)\right|_{\Gamma_{+}} \circ \phi_{+}^{-1}, \quad \mathbb{B}^{[2]}(h) w=-\left.h^{\prime}\left(\partial_{1} w\right)\right|_{\Gamma_{+}} \circ \phi_{+}^{-1}+\left.h^{-1}\left(h^{\prime}\right)^{2}\left(\partial_{2} w\right)\right|_{\Gamma_{+}} \circ \phi_{+}^{-1}$.

By Taylor expansion around $\varepsilon=0$ it is straightforward to see that there are functions

$$
\kappa^{[p]} \in C^{\infty}\left(U_{s_{1}}, H^{s_{1}-2}(\mathbb{S})\right), \quad p=0, \ldots, k+2,
$$

such that

$$
\left\|\kappa(\varepsilon, h)-\sum_{p=0}^{k+2} \varepsilon^{p} \kappa^{[p]}(h)\right\|_{s_{1}-2} \leqslant C \varepsilon^{k+3} .
$$

In particular,

$$
\kappa^{[0]}(h)=h^{\prime \prime} \quad \text { and } \kappa^{[p]}=0 \text { for } p \text { odd. }
$$

In view of (3.4), (3.5) we define

$$
\mathfrak{F}_{k}(\varepsilon, h):=\sum_{p=0}^{k+2} \varepsilon^{p-2} \sum_{j+m+l=p} \mathbb{B}^{[j]}(h) w^{[m]}(h)\left\{\kappa^{[l]}(h)\right\},
$$


$j \in\{0,2\}$. As all terms corresponding to $p=0$ and $p=1$ vanish, this is indeed a polynomial in $\varepsilon$ and

$$
\mathfrak{F}_{k} \in C^{\infty}\left([0,1) \times u_{s_{1}}, H^{s}(\mathbb{S})\right) .
$$

In particular,

$$
\mathcal{F}_{0}(\varepsilon, h)=\mathcal{F}_{k}(0, h)=\mathbb{B}^{[0]}(h) w^{[2]}(h)\left\{\kappa^{[0]}(h)\right\}+\mathbb{B}^{[2]}(h) w^{[0]}(h)\left\{\kappa^{[0]}(h)\right\}=-\left(h h^{\prime \prime \prime}\right)^{\prime}
$$

(cf. (1.3)).

It is straightforward now to obtain

$$
\left\|\mathfrak{F}(\varepsilon, h)-\mathcal{F}_{k}(\varepsilon, h)\right\|_{s} \leqslant C \varepsilon^{k+1} .
$$

To construct the approximation $h_{\varepsilon, k}$ we start with an arbitrary, sufficiently smooth, strictly positive solution $h_{0}$ of the Thin Film equation (1.3) and successively add higher order corrections. We closely follow [10, Lemma 5.3] here. Fix $T>0, h^{*} \in U_{s}$, and set for brevity

$$
\begin{aligned}
\tau & :=k+15 / 2, \\
s_{2} & :=s_{2}(k, s):=s+[k / 2](\tau-4)+\tau+1, \\
\mho_{\sigma} & :=\left\{H \in C\left([0, T], \cup_{s} \cap H^{\sigma}(\mathbb{S})\right) \cap C^{1}\left([0, T], H^{\sigma-4}(\mathbb{S})\right) \mid H(0)=h^{*}\right\}, \quad \sigma \geqslant s .
\end{aligned}
$$

Let $h_{0} \in V_{s_{2}}$ be a solution to (1.3). Observe that

$$
\mathfrak{F}_{k} \in C^{\infty}\left([0,1) \times \mathcal{U}_{\sigma}, H^{\sigma-\tau}(\mathbb{S})\right), \quad \sigma \in\left[s+\tau, s_{2}\right] .
$$

Furthermore, for $t \in[0, T]$, the linear fourth order differential operator

$$
A:=A(t):=\partial_{h} \mathcal{F}_{k}\left(0, h_{0}(t)\right)=\left[h \mapsto\left(h h_{0}^{\prime \prime \prime}(t)+h_{0}(t) h^{\prime \prime \prime}\right)^{\prime}\right]
$$

is elliptic, uniformly in $x$ and $t$.

LEMMA 4.2 Fix $h_{0}$ as above. There are positive constants $\varepsilon_{0}$ and $C$ and functions $h_{\varepsilon, k} \in V_{s+4}$, $\varepsilon \in\left[0, \varepsilon_{0}\right)$, that satisfy

$$
\int_{\mathbb{S}} h_{\varepsilon, k}(t) d x=\int_{\mathbb{S}} h_{0}(0) d x \quad \text { and } \quad\left\|\partial_{t} h_{\varepsilon, k}(t)-\mathcal{F}\left(\varepsilon, h_{\varepsilon, k}(t)\right)\right\|_{s} \leqslant C \varepsilon^{k+1}, \quad t \in[0, T] .
$$

Proof. We construct $h_{\varepsilon, k}$ by the ansatz

$$
h_{\varepsilon, k}:=h_{0}+\varepsilon h_{1}+\ldots+\varepsilon^{k} h_{k}
$$

where for $p=1, \ldots, k, h_{p}$ is recursively determined from $h_{0}, \ldots, h_{p-1}$ as solution of the fourth order linear parabolic Cauchy problem

$$
\left\{\begin{aligned}
\partial_{t} h_{p} & =\frac{1}{p^{!}} \frac{d^{p}}{d \varepsilon^{p}}\left(\left.\mathcal{F}_{k}\left(\varepsilon, h_{\varepsilon, k}\right)\right|_{\varepsilon=0}=A h_{p}+R_{p},\right. \\
h_{p}(0) & =0
\end{aligned}\right.
$$

where $R_{p}=\left[t \mapsto R_{p}(t)\right]$ is a finite sum of terms of the form

$$
\partial_{\varepsilon}^{l} \partial_{h}^{m} \mathcal{F}_{k}\left(0, h_{0}\right)\left[h_{j_{1}}, \ldots, h_{j_{m}}\right], \quad 1 \leqslant j_{i} \leqslant p-1, \quad l+\sum_{i=1}^{m} j_{i}=p .
$$


(At this point, the expression $\frac{d^{p}}{d \varepsilon^{p}}\left(\left.\mathfrak{F}_{k}\left(\varepsilon, h_{\varepsilon, k}\right)\right|_{\varepsilon=0}\right.$ should be understood in the sense of formal expansions only. It will be justified below.) Note that $\partial_{\varepsilon}^{l} \mathfrak{F}_{k}\left(0, h_{0}\right)=0$ for $l$ odd, and therefore also $h_{p}=0$ for $p$ odd. Fix $\theta \in(0,1 / 4)$. We will show by induction that

$$
h_{p} \in C^{\theta}\left([0, T], H^{\sigma_{p}}(\mathbb{S})\right) \cap C^{1+\theta}\left([0, T], H^{\sigma_{p}-4}(\mathbb{S})\right), \quad \sigma_{p}:=s_{2}-1-\frac{p}{2}(\tau-4) .
$$

For $p=0$, this follow from our assumptions by a standard interpolation argument. Suppose now this is true up to some even $p \leqslant k-2$. By (4.3) we find

$$
R_{p+2} \in C^{\theta}\left([0, T], H^{\sigma_{p}-\tau}(\mathbb{S})\right), \quad R_{p}(0) \in H^{s_{2}-\tau}(\mathbb{S})
$$

and by standard results on linear parabolic equations (cf., e.g., [12, Prop. 6.1.3])

$$
h_{p+2} \in C^{\theta}\left([0, T], H^{\sigma_{p}-\tau+4}(\mathbb{S})\right) \cap C^{1+\theta}\left([0, T], H^{\sigma_{p}-\tau}(\mathbb{S})\right) .
$$

Therefore, by our choice of $s_{2}$,

$$
h_{\varepsilon, k} \in C\left([0, T], H^{s+\tau}(\mathbb{S})\right) \cap C^{1}\left([0, T], H^{s+\tau-4}(\mathbb{S})\right) .
$$

If $\varepsilon \in\left[0, \varepsilon_{0}\right), \varepsilon_{0}$ sufficiently small, this implies $h_{\varepsilon, k}(t) \in U_{s+\tau}$, and thus, by Taylor's theorem applied at $\varepsilon=0$ to $\varepsilon \mapsto \partial_{t} h_{\varepsilon, k}-\mathcal{F}_{k}\left(\varepsilon, h_{\varepsilon, k}\right)$,

$$
\left\|\partial_{t} h_{\varepsilon, k}-\mathcal{F}_{k}\left(\varepsilon, h_{\varepsilon, k}\right)\right\|_{s} \leqslant C \varepsilon^{k+1}
$$

Consequently, we get (4.4) from this and (4.2).

Finally, for all $h \in U_{s+\tau}$ we have $\int_{\mathbb{S}} \mathcal{F}(\varepsilon, h) d x=0$, cf. [5, Lemma 3.1] and [13, Lemma 1]. Therefore, by (4.4), $\int_{\mathbb{S}} \partial_{t} h_{\varepsilon, k} d x=O\left(\varepsilon^{k+1}\right)$. This implies $\int_{\mathbb{S}} h_{p} d x=0, p=1, \ldots, k$, and thus the lemma is proved completely.

\section{Proof of the main result}

Let $T, u_{s}, h^{*}$ as in the previous section and fix $T^{\prime} \in(0, T]$. Let

$$
h_{\varepsilon} \in C\left(\left[0, T^{\prime}\right], u_{s}\right) \cap C^{1}\left(\left[0, T^{\prime}\right], H^{s-3}(\mathbb{S})\right)
$$

be a solution of (3.3) with $h_{\varepsilon}(0)=h^{*}$. For given, sufficiently smooth $h_{0}$ solving (1.3), we denote by $h_{\varepsilon, k}$ the function constructed in Lemma 4.2. The following energy estimates are the core of our result.

Proposition 5.1 (i) Fix $k \in \mathbb{N}$ and a solution $h_{0} \in V_{s_{2}(k)}$ of (1.3). There are constants $C$ and $\varepsilon_{0}$ depending on $U_{s}, k, T$, and $h_{0}$ such that

$$
\left\|h_{\varepsilon}(t)-h_{\varepsilon, k}(t)\right\|_{1} \leqslant C \varepsilon^{k+1}, \quad \varepsilon \in\left(0, \varepsilon_{0}\right), t \in\left[0, T^{\prime}\right] .
$$

(ii) Fix $n \in \mathbb{N}$. There is a $\beta=\beta(s, n) \in \mathbb{N}$ such that for any solution $h_{0} \in V_{\beta}$ to (1.3) there are constants $C$ and $\varepsilon_{0}$ depending on $U_{s}, n, T$, and $h_{0}$ such that

$$
\left\|h_{\varepsilon}(t)-h_{\varepsilon, n-1}(t)\right\|_{s} \leqslant C \varepsilon^{n}, \quad \varepsilon \in\left(0, \varepsilon_{0}\right), t \in\left[0, T^{\prime}\right] .
$$


Proof. (i) Let $\varepsilon_{0}$ be small enough to ensure that $h_{\varepsilon, k}(t) \in U_{s}, \varepsilon \in\left[0, \varepsilon_{0}\right), t \in[0, T]$.

We introduce the differences

$$
d(t):=h_{\varepsilon}(t)-h_{\varepsilon, k}(t) \quad \text { and } \quad \delta(t):=\kappa\left(\varepsilon, h_{\varepsilon}(t)\right)-\kappa\left(\varepsilon, h_{\varepsilon, k}(t)\right) .
$$

We obviously have

$$
\delta(t)=\int_{0}^{1} \kappa^{\prime}\left(\varepsilon, \tau h_{\varepsilon}(t)-(1-\tau) h_{\varepsilon, k}(t)\right)[d(t)] d \tau, \quad t \in\left[0, T^{\prime}\right],
$$

and since

$$
\kappa^{\prime}(\varepsilon, h)[d]=\left(\frac{d^{\prime}}{\left(1+\varepsilon^{2} h^{\prime 2}\right)^{3 / 2}}\right)^{\prime},
$$

we obtain that there exist positive constants $c_{1,2}=c_{1,2}\left(U_{s}\right)$ such that

$$
c_{1}\|d\|_{\sigma} \leqslant\|\delta\|_{\sigma-2} \leqslant c_{2}\|d\|_{\sigma}, \quad \sigma \in[1,2] .
$$

(Here and in the sequel, we will omit the argument $t$ if no confusion is likely.) In the same spirit, for $h, \bar{h} \in U_{s}$, we introduce the bilinear form $B(\varepsilon, h, \bar{h}): H^{1}(\mathbb{S}) \times H^{1}(\mathbb{S}) \rightarrow \mathbb{R}$ by

$$
B(\varepsilon, h, \bar{h})(e, f):=\int_{0}^{1} \int_{\mathbb{S}} \frac{e^{\prime} f^{\prime}}{\left(1+\varepsilon^{2}\left(\tau h^{\prime}+(1-\tau) \bar{h}^{\prime}\right)^{2}\right)^{3 / 2}} d \sigma d \tau .
$$

Observe that there are positive constants $c_{1,2}=c_{1,2}\left(U_{s}\right)$ such that

$$
c_{1}\|d\|_{1}^{2} \leqslant B(\varepsilon, h, \bar{h})(d, d) \leqslant c_{2}\|d\|_{1}^{2}
$$

as $d(t)$ has zero average over $\mathbb{S}$.

From (5.3) and (3.28) we find, via integration by parts,

$$
\begin{aligned}
-\left\langle\partial_{t} d \mid \delta\right\rangle_{L^{2}(\mathbb{S})}= & B\left(\varepsilon, h_{\varepsilon}, h_{\varepsilon, k}\right)\left(d, \partial_{t} d\right) \\
= & \frac{1}{2}\left(\left(\partial_{t}\left(B\left(\varepsilon, h_{\varepsilon}, h_{\varepsilon, k}\right)(d, d)\right)\right.\right. \\
& \left.\quad-\partial_{h} B\left(\varepsilon, h_{\varepsilon}, h_{\varepsilon, k}\right)(d, d) \partial_{t} h_{\varepsilon}-\partial_{\bar{h}} B\left(\varepsilon, h_{\varepsilon}, h_{\varepsilon, k}\right)(d, d) \partial_{t} h_{\varepsilon, k}\right) \\
\geqslant & \frac{1}{2} \partial_{t}\left(B\left(\varepsilon, h_{\varepsilon}, h_{\varepsilon, k}\right)(d, d)\right)-C\|d\|_{1}^{2} .
\end{aligned}
$$

Furthermore, from (3.3) and (4.4) we have

$$
\begin{aligned}
\partial_{t} d(t) & =F\left(\varepsilon, h_{\varepsilon}(t)\right)\left\{\kappa\left(\varepsilon, h_{\varepsilon}(t)\right)\right\}-F\left(\varepsilon, h_{\varepsilon, k}(t)\right)\left\{\kappa\left(\varepsilon, h_{\varepsilon, k}(t)\right)\right\}+R(t) \\
& =F\left(\varepsilon, h_{\varepsilon}(t)\right)\{\delta(t)\}+\widetilde{R}(t)+R(t),
\end{aligned}
$$

where

$$
\max _{[0, T]}\|R(t)\|_{s} \leqslant C \varepsilon^{k+1}
$$

and

$$
\widetilde{R}:=\int_{0}^{1} F^{\prime}\left(\varepsilon, \tau h_{\varepsilon, k}+(1-\tau) h_{\varepsilon}\right)[d]\left\{\kappa\left(\varepsilon, h_{\varepsilon, k}\right)\right\} d \tau
$$


By Lemma 3.6 and (5.4),

$$
\|\widetilde{R}\|_{1 / 2} \leqslant C\|d\|_{3 / 2}\left\|\kappa\left(\varepsilon, h_{\varepsilon, k}\right)\right\|_{s_{0}+3 / 2} \leqslant C\|\delta\|_{-1 / 2}, \quad \varepsilon \in\left(0, \varepsilon_{0}\right), t \in\left[0, T^{\prime}\right] .
$$

Multiplying (5.7) by $-\delta$ and applying (3.19), (5.8), (5.9), and an interpolation inequality we get

$$
\begin{aligned}
-\left\langle\partial_{t} d \mid \delta\right\rangle_{L^{2}(\mathbb{S})} & \leqslant-c\|\delta\|_{1 / 2, \varepsilon}^{2}+C\|\delta\|_{-1 / 2}^{2}+C \varepsilon^{k+1}\|\delta\|_{-1} \\
& \leqslant-c\|\delta\|_{0}^{2}+\left(c\|\delta\|_{0}^{2}+C\|\delta\|_{-1}^{2}\right)+C\left(\varepsilon^{2 k+2}+\|\delta\|_{-1}^{2}\right) \\
& \leqslant C\left(\|\delta\|_{-1}^{2}+\varepsilon^{2 k+2}\right) .
\end{aligned}
$$

Together with (5.6), this shows that

$$
\frac{d}{d t} B\left(\varepsilon, h_{\varepsilon}, h_{\varepsilon, k}\right)(d, d) \leqslant C\left(\varepsilon^{2 k+2}+B\left(\varepsilon, h_{\varepsilon}, h_{\varepsilon, k}\right)(d, d)\right)
$$

for all $\varepsilon \in\left(0, \varepsilon_{0}\right), t \in\left[0, T^{\prime}\right]$. Taking into consideration that $d(0)=0$, we find by Gronwall's inequality that

which proves (5.1).

$$
c_{1}\|d\|_{1}^{2} \leqslant B\left(\varepsilon, h_{\varepsilon}, h_{\varepsilon, k}\right)(d, d) \leqslant C(T) \varepsilon^{2 k+2},
$$

(ii) Set $k:=n+5 s-1$ and $\beta:=s_{2}(k)$. Let $\varepsilon_{0}$ be small enough to ensure that $h_{\varepsilon, k}(t) \in U_{s}$, $\varepsilon \in\left[0, \varepsilon_{0}\right), t \in[0, T]$.

Instead of (5.2) we are going to prove the equivalent estimate

$$
\left\|h_{\varepsilon}(t)-h_{\varepsilon, k}(t)\right\|_{s} \leqslant C \varepsilon^{n}, \quad \varepsilon \in\left(0, \varepsilon_{0}\right), t \in\left[0, T^{\prime}\right] .
$$

Let $\delta_{s-1}:=\kappa^{\prime}\left(\varepsilon, h_{\varepsilon}\right)\left[d^{(s-1)}\right]$. Then, in analogy to (5.6),

$$
-\left\langle\partial_{t} d^{(s-1)} \mid \delta_{s-1}\right\rangle_{L^{2}(\mathbb{S})} \geqslant \frac{1}{2} \partial_{t}\left(B\left(\varepsilon, h_{\varepsilon}\right)\left(d^{(s-1)}, d^{(s-1)}\right)\right)-C\|d\|_{s}^{2},
$$

where $B\left(\varepsilon, h_{\varepsilon}\right):=B\left(\varepsilon, h_{\varepsilon}, h_{\varepsilon}\right)$.

On the other hand, differentiating the relation

$$
\partial_{t} d=\mathfrak{F}\left(\varepsilon, h_{\varepsilon}\right)-\mathcal{F}\left(\varepsilon, h_{\varepsilon, k}\right)+R
$$

$(s-1)$ times with respect to $x$ we get (cf. Lemma 3.8)

$$
\partial_{t} d^{(s-1)}=-F\left(\varepsilon, h_{\varepsilon}\right)\left[\delta_{s-1}\right]+P_{s}\left(\varepsilon, h_{\varepsilon}, h_{\varepsilon, k}\right)+R^{(s-1)} .
$$

Recalling (3.19), (3.34), (5.4), and (5.8) we obtain from this by Young's inequality

$$
\begin{aligned}
-\left\langle\partial_{t} d^{(s-1)} \mid \delta_{s-1}\right\rangle_{L^{2}(\mathbb{S})} & \leqslant-c\left\|\delta_{s-1}\right\|_{1 / 2, \varepsilon}^{2}+C \varepsilon^{-2}\|d\|_{s+1 / 2}\left\|\delta_{s-1}\right\|_{1 / 2}+C \varepsilon^{k+1}\left\|\delta_{s-1}\right\|_{-1} \\
& \leqslant-c\left\|\delta_{s-1}\right\|_{0}^{2}+C \varepsilon^{-5}\|d\|_{s+1 / 2}^{2}+C \varepsilon^{2 k+2}+\left\|\delta_{s-1}\right\|_{-1}^{2} \\
& \leqslant-c\|d\|_{s+1}^{2}+C \varepsilon^{-5}\|d\|_{s+1 / 2}^{2}+C \varepsilon^{2 k+2} .
\end{aligned}
$$

Consequently, by (5.11), (5.1), and an interpolation inequality,

$$
\begin{aligned}
\partial_{t} B\left(\varepsilon, h_{\varepsilon}\right)\left(d^{(s-1)}, d^{(s-1)}\right) & \leqslant-c \mid d\left\|_{s+1}^{2}+C \varepsilon^{-5}\right\| d\left\|_{s+1}^{2-1 / s}\right\| d \|_{1}^{1 / s}+C \varepsilon^{2 k+2} \\
& \leqslant C\left(\varepsilon^{-10 s}\|d\|_{1}^{2}+\varepsilon^{2 k+2}\right) \leqslant C \varepsilon^{2 k+2-10 s} \leqslant C \varepsilon^{2 n}
\end{aligned}
$$

Integrating over $t$ and using (5.5), we obtain (5.10). 
Proof of Theorem 1.2. Choose $k$ and $\beta=\beta(s, n)$ as in the proof of Proposition 5.1 (ii), let $h^{*}:=h_{0}(0)$ and let $\alpha$ and $M$ be such that $h_{0}([0, T]) \subset U_{s}$. By compactness, we have $\mu:=\operatorname{dist}\left(\partial U_{s}, h_{0}([0, T])\right)>0$. Let $\varepsilon_{0}$ be small enough to ensure that $h_{\varepsilon, k}([0, T]) \subset U_{s}$,

$$
\operatorname{dist}\left(\partial u_{s}, h_{\varepsilon, n-1}([0, T])\right)>\mu / 2, \quad \varepsilon \in\left[0, \varepsilon_{0}\right),
$$

and $C \varepsilon_{0}^{n}<\mu / 4$, where $C$ is the constant from (5.2).

Let $\varepsilon \in\left(0, \varepsilon_{0}\right)$ and let

$$
h_{\varepsilon} \in C\left(\left[0, T_{\varepsilon}\right), H^{\beta-1}(\mathbb{S})\right) \cap C^{1}\left(\left[0, T_{\varepsilon}\right), H^{\beta-4}(\mathbb{S})\right)
$$

be a maximal solution to (3.3) with $h_{\varepsilon}(0)=h^{*}$. In view of Proposition 5.1 (ii), it remains to show that $T_{\varepsilon}>T$. Assume $T_{\varepsilon} \leqslant T$. The blowup result in Theorem 1.1 (iii) implies that there is a $T^{\prime} \in(0, T)$ such that $h_{\varepsilon}\left(\left[0, T^{\prime}\right]\right) \subset U_{s}$ but dist $\left(\partial U_{s}, h\left(T^{\prime}\right)\right)<\mu / 4$. In view of (5.2) and (5.12), this is a contradiction to our choice of $\varepsilon_{0}$.

Acknowledgements. The research leading to this paper was carried out in part while the second author enjoyed the hospitality of the Institute of Applied Mathematics as a guest researcher at Leibniz University Hannover. Moreover, we express our gratitude to Prof. M. Günther (Leipzig University) whose ideas for [10] are crucial for the present paper as well.

\section{REFERENCES}

1. Bazaliy, B. \& Friedman, A., The Hele-Shaw problem with surface tension in a half-plane, $J$. Differential Equations 216 (2005), 439-469. Zb11076.76028 MR2162342

2. Constantin, P. ET AL., Droplet breakup in a model of the Hele-Shaw cell, Physical Review E 47 (1993), 4169-4181. MR1377907

3. Dupont, T. F. ET AL., Finite-time singularity formation in Hele-Shaw systems, Phys. Rev. E 47(6) (1993), 4182-4196. MR1377908

4. Elliott, C. M. \& OCKendon, J. R., Weak and variational methods for moving boundary problems, Pitman 1982. Zb10476.35080 MR0650455

5. Escher, J. \& Matioc, B.-V., On periodic Stokesian Hele-Shaw flows with surface tension, European J. Appl. Math. 19 (2008), 717-734. Zbl1148.76020 MR2463227

6. Escher, J. \& MATioc, B.-V., Existence and stability results for periodic Stokesian Hele-Shaw flows, SIAM J. Math. Anal. 40 (2008/09), 1992-2006. Zbl1176. 35181 MR2471909

7. Escher, J. \& Prokert, G., Analyticity of solutions to nonlinear parabolic equations on manifolds and an application to Stokes flow, J. Math. Fluid Mech. 8 (2006), 1-35. Zbl1102. 35048 MR2205149

8. Escher, J. \& Simonett, G., Classical solutions for Hele-Shaw models with surface tension, Advances in Diff. Eq. 2 (1997), 619-642. Zbl1023. 35527 MR1441859

9. Giacomelli, L. \& OTto, F., Rigorous lubrication approximation, Interfaces and Free Boundaries 5 (2003), 483-529. Zbl1039.76012 MR2031467

10. GÜNTher, M. \& Prokert, G., A justification for the thin film approximation of Stokes flow with surface tension, J. Differential Equations 245 (2008), 2802-2845. Zbl pre05365153 MR2454804

11. Hulshof, J., Some aspects of the thin film equation, Proceedings of European Congress of Mathematics Vol. II, 291-301, Birkhäuser 2001. Zbl1027 . 35056 MR1905370

12. LunARd, A., Analytic semigroups and optimal regularity in parabolic problems, Birkhäuser 1995. Zb10816.35001 MR1329547

13. Prokert, G., Existence results for Hele-Shaw flow driven by surface tension, European J. Appl. Math. 9 (1998), 195-221. Zbl0919.35005 MR1630665 